\title{
Joint Radio Resource Management and Link Adaptation for Multicasting 802.11ax-based WLAN Systems
}

\author{
Vu Nguyen Ha, Member, IEEE, Georges Kaddoum, Senior Member, IEEE, \\ and Gwenael Poitau
}

\begin{abstract}
Adopting OFDMA and MU-MIMO techniques for both downlink and uplink IEEE 802.11ax will help nextgeneration WLANs efficiently cope with large numbers of devices but will also raise some research challenges. One of these is how to optimize the channelization, resource allocation, beamforming design, and MCS selection jointly for IEEE 802.11ax-based WLANs. In this paper, this technical requirement is formulated as a mixed-integer non-linear programming problem maximizing the total system throughput for the WLANs consisting of unicast users with multicast groups. A novel two-stage solution approach is proposed to solve this challenging problem. The first stage aims to determine the precoding vectors under unit-power constraints. These temporary precoders help re-form the main problem into a joint power and radio resource allocation one. Then, two low-complexity algorithms are proposed to cope with the new problem in stage two. The first is developed based on the well-known compressed sensing method while the second seeks to optimize each of the optimizing variables alternatively until reaching converged outcomes. The outcomes corresponding to the two stages are then integrated to achieve the complete solution. Numerical results are provided to confirm the superior performance of the proposed algorithms over benchmarks.
\end{abstract}

Index Terms-IEEE 802.11ax, WLAN, OFDMA, channelization, resource allocation, MU-MIMO, link adaptation, multicast transmission.

\section{INTRODUCTION}

$\mathbf{R}$ ECENTLY, it has been foreseen that, in the near future, emerging wireless networks will face an unprecedented increase in traffic volume corresponding to various types of services and use cases [1]. To meet such ever-growing demands, together with $5 \mathrm{G}$, the sixth generation of wireless local access networks (WLANs) are expected to support a large number of wireless connections from different applications with diverse requirements including massive machine type communications (mMTC) and enhanced mobile broadband

Manuscript received June 29, 2020; revised January 14, 2021; and accepted March 29, 2021. This work was supported by the ULTRA TCS research chair on intelligent tactical wireless networks for challenging environments, and by the grant number CRDPJ 538896-19 from the National Natural Sciences and Engineering Research Council of Canada (NSERC). The associate editor coordinating the review of this paper and approving it for publication was K. R. Chowdhury.

$\mathrm{Vu} \mathrm{N}$. Ha and G. Kaddoum are with the Resilient Machine learning Institute (ReMI) of the École de Technologie Supérieure (ÉTS), University of Québec, Montréal, Canada (e-mail: vu.ha-nguyen.1@ens.etsmtl.ca; Georges.Kaddoum@etsmtl.ca).

G. Poitau is with the Resilient Machine learning Institute (ReMI) and Ultra Electronics TCS, Montréal, Canada (e-mail: Gwenael.Poitau@UltraTCS.com).
TABLE I

RESOURCE UNIT FORMAT IN WIFI 802.11 AX.

\begin{tabular}{|l|l|l|l|l|}
\hline RU type & $\mathbf{2 0} \mathbf{~ M H z}$ & $\mathbf{4 0} \mathbf{~ M H z}$ & $\mathbf{8 0} \mathbf{~ M H z}$ & $\mathbf{1 6 0} \mathbf{~ M H z}$ \\
\hline \hline 26-tone $(*)-\ell=5$ & 9 & 18 & 37 & 74 \\
\hline 52-tone $-\ell=4$ & $4+1^{*}$ & $8+2^{*}$ & $16+5^{*}$ & $32+10^{*}$ \\
\hline 106-tone $-\ell=3$ & $2+1^{*}$ & $4+2^{*}$ & $8+5^{*}$ & $16+10^{*}$ \\
\hline 242-tone $-\ell=2$ & 1 & 2 & $4+1^{*}$ & $8+2^{*}$ \\
\hline 484-tone $-\ell=1$ & NA & 1 & $2+1^{*}$ & $4+2^{*}$ \\
\hline 996-tone $-\ell=0$ & NA & NA & 1 & 2 \\
\hline \multicolumn{5}{|c|}{ (* indicates 26-tone RU). }
\end{tabular}

(eMBB) in the new Internet of Thing era [1], [2]. Various technological concepts have been developed for radio access, network resource management, and performance improvement in wireless systems which have been standardized in IEEE 802.11ax amendment for next-generation WLANs [3]. Specifically, the IEEE 802.11ax standard includes the orthogonal frequency-division multiple access (OFDMA) and multi-user multiple- input and multiple-output (MU-MIMO). Adopting OFDMA, IEEE 802.11ax subdivides the channel bandwidth into a number of tones with smaller sub-carrier spacing, e.g., $\Delta f_{\mathrm{ax}}=78.125 \mathrm{kHz}$, four times smaller than what employed in legacy WiFi standards (IEEE $802.11 \mathrm{~g} / \mathrm{n} / \mathrm{ac}$ ) which can increase the efficiency of frequency utilization by squeezing more tones nearby the direct current and edge tones. Another significant improvement in IEEE 802.11ax is that the tones can be grouped into a combination of different types of resource units (RUs) - as in Table I] and these RUs can be allocated to various users for simultaneous communication [3], [5]. In addition, IEEE 802.11ax also adopts MU-MIMO technology for both downlink and uplink transmission. Acquiring the OFDMA and MU-MIMO technologies allows the WLANs to serve the higher number of devices and services in one transmission frame [5], [6]. Like in legacy WiFi systems, dynamic link adaptation (LA) functionality also plays an important role in IEEE 802.11ax to cope with heterogeneous services with different required data rate. Specially, IEEE 802.11ax has added two new options, i.e., the 1024-QAM with two code rates, $3 / 4$ and $5 / 6$, into the modulation and coding scheme (MCS) table [5] which can help next-generation WLANs enhance the good channels in some specific scenarios, e.g., residential indoor or offices.

Embracing OFDMA and MU-MIMO technologies and enlarging the MCS table, 802.11ax-based WLANs are expected to support a larger number of devices and heterogeneous services with a larger range of required data rates [5], [6]. In 
these schemes, the broadcast, unicast, and multicast services with various applications, such as ultra high-definition (HD) streaming, HD video conferencing, and augmented reality $(\mathrm{AR}) /$ virtual reality (VR)/ mixed reality (MR), etc. can be served concurrently [7]. Together with unicast transmission, which is a tradition use case in WLANs, multicast communication is one of WLANs' relevant usage scenarios since it can provide high spectrum efficiency to WLAN through delivering a large amount of data (e.g., real-time audio and video) to a group of multicast devices over one RU simultaneously [7][9]. Hence, balancing the unicast and multicast transmission is also an interesting research issue in the heterogeneous wireless systems [7]. For instance, WLANs of sport-event stadiums serving thousands spectators are expected to be able multicast videos of the match, the highlights, or real-time push notifications to to specific groups of users, and provide the traditional unicast transmission to others concurrently [9], [47]. With MU-MIMO, the multicast transmission can increase the network throughput significantly by carefully optimizing the precoding vectors regarding the channels between the AP and all clients [10]. Besides, OFDMA technology can help next-generation WLANs manage the unicast and multicast transmission more efficiently thanks to the flexible channelization and RU assignment strategies. Therefore, effectively utilizing the advantages of OFDMA and MU-MIMO using intelligent radio resource management (RRM) mechanisms for 802.11ax-based WLAN systems supporting both unicast and multicast services is an interesting and demanded research problem which will be studied in this paper.

\section{A. Related Works}

The RRM mechanisms for 802.11ax WLANs systems have been studied extensively in the literature where most of the existing works consider, separately or jointly OFDMA and MU-MIMO, in their designs. Specifically, Lee et al. in [11] have presented a new hybrid multiple access coordination scheme (HMAC) which can increase the spectrum efficiency of the OFDMA WLANs. Based on a centralized access controller, HMAC allows multiple devices to function simultaneously in order to maximize channel utilization. Regarding URLLC applications, the work in [12] proposed an OFDMA RRM algorithm for 802.11ax-based WLANs which scarifies the throughput insignificantly to maintain the transmission delay lower than one millisecond and the reliability higher than $99.999 \%$. In another approach, three user-scheduling frameworks corresponding to three design metrics, i.e. sumrate maximization, fairness, and the shortest remaining process time were proposed for IEEE 802.11ax-based WLAN in [13] to efficiently improve the usage of OFDMA RUs. However, the RRM strategies studied in these three works [11]-[13] have been developed without integration with channelization, LA, and MU-MIMO transmission. Considering the joint OFDMA and MU-MIMO design for 802.11ax based WLANs, Wang et al. in [14] focused on how to split the channel into a combination of RUs and how to assign these RUs to users and groups of MU-MIMO users to maximize the sum rate. A greedy design and an efficient recursive algorithm were then proposed to achieve the design goal; however, the MU-MIMO transmission is not optimized in this work. Another novel multi-user transmission scheme for 802.11ax-based WLANs was proposed in [15] which enhances the advantages of both OFDMA and MU-MIMO for the system utility maximization. Specifically, this framework exploited the information from uplink transmission for optimizing the user-scheduling and RRM solution for downlink side.

The LA designs for 802.11ax-based WLANs have been also considered in literature; however, the number of related works is very limited. In particular, [16] has utilized a software define network (SDN) approach to propose a link adaptation framework for next-generation WLANs which can dynamically support numerous devices with various degrees of Quality of Service (QoS) or Quality of Experience (QoE) requirements. In this work, an SDN agent was incorporated with application program interfaces to separate the control and data plane based on which an on-line RRM mechanism was developed by providing the abstraction of wireless elements to the SDN controller. Employing reinforcement learning tools, [17] has proposed an intelligent user-scheduling and link adaptation framework which can dynamically provide MCS selection and user scheduling for MU-MIMO transmission at the beginning of every periodic interval. Regarding multicast transmission, the authors in [18] developed a link adaptation mechanism for video streaming $\mathrm{WiFi}$ systems. In this mechanism, the controller was implemented for continuously tracking the packet-transmission performance corresponding to every selected MCS index based on which the most efficient MCSs are dynamically determined for the next transmission. However, the scenario with coexisting unicast and multicast transmission was not studied in this work.

\section{B. Contributions and Organization of the Paper}

To the best of our knowledge, the joint design of multicast MU-MIMO transmission along with the channelization, RU assignment, and LA for 802.11ax-based WLANs supporting coexisting unicast and multicast services has not been considered in the literature. This paper is the first work tackling this subject which aims to maximize the total throughput. To do so, an overall two-stage solution approach and two low-complex joint RRM and LA algorithms are proposed. In particular, the main contributions of this paper are summarized as follows:

- We approximate the block error rate (BLER) of the transmission performed over an RU with a specific MCS and low-density parity-check (LDPC) encoder to an exponential approximation function of the effective signal-to-noise (SNR) value - a special average value representing the SNRs over all tones in one RU [22]. The function can be calibrated for each MCS setting through calibrating parameters. Then, Monte-Carlo simulations are implemented to collect the BLERs corresponding to 12 MCSs over different SNR values. The collected BLER data obtained from the simulations are utilized to estimate the calibrating parameters corresponding to all $12 \mathrm{MCSs}$ through the "curve-fitting" tool of Matlab.

- Employing the approximate BLER functions and taking into account the strict practical requirements of the IEEE 
802.11ax standard, we formulate the joint beamforming, RRM and LA (JRRMLA) design as a mixed-integer non-linear programming (MINLP) problem which aims to maximize the total throughput of an 802.11ax-based WLAN serving both unicast and multicast users. In this design, the single user-MIMO (SU-MIMO) transmission is optimized for each unicast user while the MU-MIMO is employed for all users of each multicast group. This MINLP problem is well-known to be NP-hard.

- To deal with this challenging problem, we propose a twostage solution approach. In stage one, the directions of the precoders are estimated by optimizing the beamforming transmission over each RU to maximize the throughput under a $1 \mathrm{~W}$ power transmission constraint. Using the temporary precoders obtained in stage one and presenting new power allocation variables, we transfer the main problem into a new one focusing on optimizing the joint design of power control, RMM, and LA. Thus, stage two aims to solve this new problem to optimize the channelization, RU assignment, and MCS selection while the complete precoding vectors are defined based on the outcomes of stage one and the power allocation results.

- To cope with the problem in stage two, we propose two efficient algorithms. In the first one, namely "compressed sensing-based relaxation algorithm”, we employ the well-known compressed sensing (CS) method to approximate the binary RU assignment variables into a linear form with specific weights. These weights will be updated iteratively. Based on this, the MINLP problem is simplified into a convex form in each iteration. Herein, the MCS variables are also updated to maximize the total throughput at the end of every iteration. Then, the solution can be obtained by iteratively updating the weights and solving the corresponding relaxed problem. In the second approach, referred to as "alternative radio resource management algorithm", we alternatively update the power allocation, MCS selection, and RU assignment while keeping the other design factors fixed.

- We also present a greedy algorithm as the referencing benchmark. This algorithm is developed based on the joint channelization and RU assignment framework given in [14]. Extensive numerical results showing the convergence and efficiency of the proposed solution approaches on the total throughput are presented. For comparison purposes, the near-optimal solution achieved by employing the supercomputer of NEOS system as well as the numerical results of the referencing algorithm and other schemes with fixed MCS selection, and only supporting unicast transmission are also brought up. The simulation results confirm the superior performance achievable by our proposed JRRMLA mechanisms for 802.11ax-based WLAN systems supporting coexisting unicast and multicast users.

The remainder of this paper is organized as follows. Section III introduces the system model, the LA mechanism, OFDM-MIMO transmission, and the problem formulation. Section III presents the two-stage solution framework and

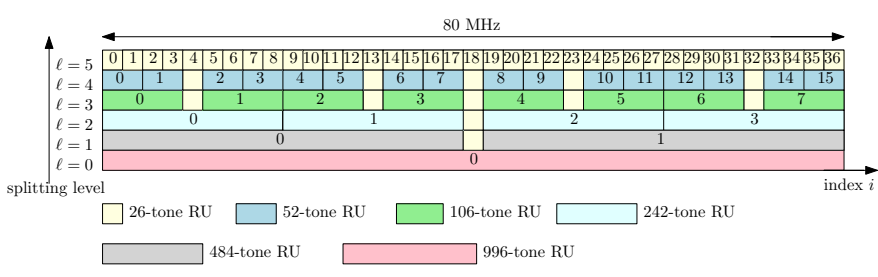

Fig. 1. OFDMA resource units for $80 \mathrm{MHz}$ channels.

the temporary precoder designs of stage one. In Section IV we propose two efficient low-complexity JRRMLA algorithms for solving the problem in stage two. Section $\mathrm{V}$ provides the complexity analysis of our proposed algorithms and introduces the greedy algorithm for comparison purposes. Section VI evaluates the performance of the proposed algorithms which is followed by Section VII concluding this work. Key notations used in the paper are summarized in Table $\mathrm{V}$.

\section{SySTEM MODEL}

In this paper, we focus on a downlink single basic service set (BSS) IEEE 802.11ax WLAN system, in which an AP operates the OFDMA $80 \mathrm{MHz}$ channel to provide services to $N^{\mathrm{UU}}$ unicast users (UUs) and $N^{\mathrm{MU}}$ multicast users (MUs) divided in $N^{\mathrm{MG}}$ multicast groups (MGs). Denote $\mathcal{U}_{n}^{\text {mul }}$ as the set of MUs in MG $n$. The same information data is processed for a specific MG $n$ and then is broadcasted to all MUs in $\mathcal{U}_{n}^{\text {mul }}$ over the assigned channel. For convenience, let $\mathcal{U}^{\mathrm{UU}}=\left\{1, \ldots, N^{\mathrm{UU}}\right\}$ and $\mathcal{G}^{\mathrm{MG}}=\left\{N^{\mathrm{UU}}+1, \ldots, N^{\mathrm{UU}}+N^{\mathrm{MG}}\right\}$ be the sets of UUs and MGs, respectively. We assume that the AP knows all the information about the users at the begin of the transmission time slot which includes the users' data and the channel state information (CSI). The CSI can be estimated by the AP based on the uplink signals from the users during the channel sounding phase [3]. Moreover, we also presume that the CSI remains unchanged during a transmission time slot and a full-buffer traffic model is considered for all users' data. Once the MGs and UUs are scheduled for downlink transmission, the AP needs to win a transmit opportunity (TXOP) for the entire frame exchange by contending for the medium [3]. After having the right to access the channel, it decides the channelization, the RU assignment, the precoding vectors, and the MCS selections for MGs/UUs' transmission. Then, the AP sends a multi-user request-to-send (MU-RTS) frame - an extended trigger frame - which consists of an RU assignment map and per-user content fields indicating the assigned RU index, the selected modulation and coding rate to the users [3], [43]. This MU-RTS frame ensures that other unscheduled users remain idle and also helps the scheduled users synchronize uplink clear-to-send (CTS) responses. After receiving the CTS messages, the AP transmits the user's data with optimized precoding vectors, MCS selections over the corresponding assigned RUs. Here, we further assume that the MU-RTS frame is correctly received by all users in this single BSS system.

\section{A. OFDMA Channelization and Resource Units Allocation}

Consider an $80 \mathrm{MHz}$ channel consisting of a total of 1024 $78.125 \mathrm{kHz}$-spacing tones. According to the IEEE 802.11ax 
standard, this channel can be divided into different types of RUs, e.g., 26-tone, 52-tone, 106-tone, 242-tone, 484-tone, and 996-tone RUs [3]. As illustrated in Fig. 1, the channel can be split 5 times. In particular, for splitting levels 1 and 2, the channel bandwidth can be divided into two 484-tone RUs or four 242-tone RUs and one 26-tone RU. For splitting levels 3 and 4, we can get 8 106-tone RUs or 16 52-tone RUs and 5 26-tone RUs. For splitting level 5, there are 37 26-tone RUs. For simplification, we name the RUs based on the splitting level and their positions as $R U(\ell, i)$ where $\ell$ is the splitting level and $i$ is the position. In addition, one denotes $\mathcal{S}_{(\ell, i)}$ as the set of all tones corresponding to $R U(\ell, i)$. The number of tones in $\mathcal{S}_{(\ell, i)}$ is defined as $S_{(\ell, i)}$ which can be found in the first column of Table [1] For different communication scenarios, the channel can be split into different combinations of RUs which is also called "channelization" [3]. For instance, the combination of $[R U(1,0), R U(5,18), R U(1,1)]$ can be exploited for three-user simultaneous transmission. When the AP needs to serve 4 users at the same time, $R U(1,0)$ can be further divided into $R U(2,0)$ and $R U(2,1)$ to achieve a combination of 4 RUs such as $[R U(2,0), R U(2,1), R U(5,18), R U(1,1)]$.

To prevent the cross interference, we assume that the RUs are channelized and assigned to the users such that there is no overlap between users' corresponding spectrum bands. Regarding this condition, we first introduce $a_{\ell, i}^{v}$ as a binary RUassignment variable corresponding to UU/MG $v$ and $R U(\ell, i)$. In particular, $a_{\ell, i}^{v}=1$ if $R U(\ell, i)$ is assigned to UU/MG $v$, i.e, $v \in \mathcal{G} \mathcal{U}=\mathcal{U}^{\cup U} \cup \mathcal{G}^{\mathrm{MG}}$, and vice versa. We further denote $\mathbf{b}_{\ell, i}=\left[b_{\ell, i}^{0}, \ldots, b_{\ell, i}^{36}\right] \in \mathbb{R}^{1 \times 37}$ as the indicating vector which can represent the overlap between $R U(\ell, i)$ and the 26-tone RUs. The elements of $\mathbf{b}_{\ell, i}$ are defined as follows,

$$
b_{\ell, i}^{n}= \begin{cases}1, & \text { if } R U(\ell, i) \text { overlaps } R U(5, n), \\ 0, & \text { otherwise. }\end{cases}
$$

For example, vector $\mathbf{b}_{0,0}=[1, \ldots, 1]$ indicates that $R U(0,0)$ overlaps all 37 26-tone RUs while $\mathbf{b}_{3,0}=[1,1,1,1,0, \ldots, 0]$ implies that $R U(3,0)$ only overlaps the first four 26-tone RUs. As can be seen, the spectrum overlap will not occur if there is no 26-tone RU that is conquered by two or more assigned RUs. Then, this assignment condition can be transferred into the following constraint,

$$
\sum_{\forall(\ell, i)} \sum_{v \in \mathcal{G} \mathcal{U}} a_{\ell, i}^{v} \mathbf{b}_{\ell, i} \leq \mathbf{1}^{1 \times 37}
$$

where $\mathcal{G} \mathcal{U}=\mathcal{U}^{\mathrm{UU}} \cup \mathcal{G}^{\mathrm{MG}}$ represents the set of all UUs and MGs. In this paper, the AP will assign RUs to UUs/MGs in a fair manner such that every scheduled user must be served, i.e., every user is assigned only one RU according to the resource allocation regulation of IEEE 802.11ax amendment [3]. Additionally, we only focus on feasible schemes where the number of UUs/MGs is less than 37 - the highest number of RUs which can be channelized as shown in Fig. 1. The infeasible scenarios where the number of UUs/MGs is larger than 37 can be handled by employing user-scheduling mechanisms. Such schemes are out of the scope of this paper and will be considered in future works.
TABLE II

MCS TABLE EMPLOYED IN WIFI 802.11AX SYSTEMS.

\begin{tabular}{|c|c|c|c|c|}
\hline $\begin{array}{c}\text { index } \\
m\end{array}$ & Modulation & $\begin{array}{c}\text { bits/symbol } \\
r_{\mathrm{C}}(m)\end{array}$ & $\begin{array}{c}\text { Coding Rate } \\
c_{\mathrm{cr}}(m)\end{array}$ & $r_{\mathrm{C}}(m) c_{\mathrm{cr}}(m)$ \\
\hline 0 & BPSK & 1 & $1 / 2$ & $1 / 2$ \\
\hline 1 & QPSK & 2 & $1 / 2$ & 1 \\
\hline 2 & QPSK & 2 & $3 / 4$ & $3 / 2$ \\
\hline 3 & 16-QAM & 4 & $1 / 2$ & 2 \\
\hline 4 & 16-QAM & 4 & $3 / 4$ & 3 \\
\hline 5 & 64-QAM & 8 & $2 / 3$ & $16 / 3$ \\
\hline 6 & 64-QAM & 8 & $3 / 4$ & 6 \\
\hline 7 & 64-QAM & 8 & $5 / 6$ & $20 / 3$ \\
\hline 8 & 256-QAM & 16 & $3 / 4$ & 12 \\
\hline 9 & 256-QAM & 16 & $5 / 6$ & $40 / 3$ \\
\hline 10 & 1024-QAM & 32 & $3 / 4$ & 24 \\
\hline 11 & 1024-QAM & 32 & $5 / 6$ & $80 / 3$ \\
\hline
\end{tabular}

\section{B. Link Adaptation}

The IEEE 802.11ax amendment has added an optional 1024QAM modulation to the legacy options of BPSK, 16-QAM, 64-QAM, and 256-QAM to support the transmission with very good channel conditions - i.e., high signal-to-noise ratios (SNRs). Together with forward error correction (FEC) codes, the specific MCS table for 802.11ax-based WLAN, given in Table II], has been updated [4]. Let $c_{\mathrm{C}}(m)$ and $r_{\mathrm{cr}}(m)$ be the modulated bits per symbol and the channel coding rate corresponding to MCS index $m$, and $m^{v}$ be the MCS index selected for the transmission corresponding to UU/MG $v$. For MCS $m^{v}$, the throughput of user $\mathrm{X}$, which stands for UU $v$ $(\mathrm{x}:=v)$ or user $k$ in $\mathrm{MG} v(\mathrm{x}:=(v, k))$, over the $R U(\ell, i)$ can be calculated as [19]

$$
\begin{aligned}
& T_{\ell, i}^{\mathrm{x}}\left(m^{v}, \gamma_{\ell, i}^{\mathrm{x}}\right) \\
& \quad=\frac{L^{v}}{L^{v}+O^{v}} S_{(\ell, i)} c_{\mathrm{C}}\left(m^{v}\right) r_{\mathrm{cr}}\left(m^{v}\right)\left[1-B L E R\left(m^{v}, \gamma_{\ell, i}^{\mathrm{x}}\right)\right](3)
\end{aligned}
$$

where $L^{v}$ and $O^{v}$ are the packet and the overhead lengths (in bits) corresponding to the transmission of UU/MG $v$ and $\operatorname{BLER}\left(m^{v}, \gamma_{\ell, i}^{\mathrm{x}}\right)$ is the block error rate (BLER) corresponding to the data transmission with packet length $L^{v}$ for given MCS index $m^{v}$ and SNR $\gamma_{\ell, i}^{\mathrm{x}}$.

To the best of our knowledge, there is no closed-form equation for the $B L E R\left(m^{v}, \gamma_{\ell, i}^{\mathrm{x}}\right)$. But some works have reported that the BLER strongly depends on the coding/decoding technique 1 the computation effort, and the number of decoding iterations employed at the receiver [20], [44]. For given $L^{v}$, Park et al. in [21] have approximated the BLER corresponding to a specific MCS index as a function of the average SNR as follows

$$
\operatorname{BLER}(m, \gamma)=e^{-\alpha(m)(\gamma-\beta(m))},
$$

where $\alpha(m)$ and $\beta(m)$ are constant parameters representing the slope value and dropping point corresponding to MCS index $m$. These parameters correspond to a specific decoding technique applied at the receiver, which can be estimated based on the practical simulation results. The function given in 4

\footnotetext{
${ }^{1}$ The coding performance mainly depends on the type of employed LDPC technique, such as regular or irregular LDPC, and the number of ones in the coding matrix.
} 
yields the approximate form of the throughput of user $\mathrm{x}$ as follows

$$
T_{\ell, i}^{\mathrm{x}}\left(m^{v}, \gamma_{\ell, i}^{\mathrm{x}}\right)=\theta_{\ell, i}^{v}\left(m^{v}\right)\left(1-A\left(m^{v}\right) e^{\left.-\alpha\left(m^{v}\right) \gamma_{\ell, i}^{\mathrm{x}}\right)},\right.
$$

where $\theta_{\ell, i}^{v}\left(m^{v}\right)=L^{v} /\left(L^{v}+O^{v}\right) S_{(\ell, i)} c_{\mathrm{C}}\left(m^{v}\right) r_{\mathrm{cr}}\left(m^{v}\right)$ and $A\left(m^{v}\right)=e^{\alpha\left(m^{v}\right) \beta\left(m^{v}\right)}$.

\section{MIMO-OFDM transmission with given MCS}

We further assume a MIMO transmission in this system where the AP is equipped with $N_{\mathrm{T}}$ antennas while single antennas are employed at the users. Assuming that $R U(\ell, i)$ is allocated to UU/MG $v$, the following briefly overviews the signal-processing operations corresponding to the MIMOOFDM transmission for UU/MG $v$ over $R U(\ell, i)$.

Over an occupied RU, a packet of information bits is transmitted through frames. Each frame is composed of $L^{v}$ coded OFDM symbols and $S_{(\ell, i)}$ tones corresponding to $R U(\ell, i)$. The information bits are first encoded at a LDPC encoder with a coding rate of $c_{\mathrm{cr}}\left(m^{v}\right)$. The encoded bits are then modulated. In this process, the bits are divided so that every identical symbol corresponding to every tone will carry $r_{\mathrm{C}}\left(m^{v}\right)$ bits. A frame of $r_{\mathrm{C}}\left(\mathrm{m}^{v}\right)$ bits, which are modulated into one such symbol, is so-called code-word. Allocated bits for each tone are independent of the OFDM symbol index. In particular, the code-words are interleaved by a random block bit-level interleaver with a depth of $S_{(\ell, i)} r_{\mathrm{C}}\left(m^{v}\right)$ and are then Graymapped into one of the $2^{r_{c}\left(m^{v}\right)}$ complex-valued symbols.

Let $s_{t}^{v}$ be the transmitted symbol corresponding to UU/MG $v$ and tone $t$, then symbol $s_{t}^{v}$ is multiplied by an $N_{\mathrm{T}} \times 1$ precoding vector before being transmitted to UU/MG $v$. Denote $\mathbf{w}_{\ell, i, t}^{v}$ as the precoding vector designed for UU/MG $v$ over tone $t$ of $R U(\ell, i)$. The received signal at user $\mathrm{x}$ (standing for UU $v$ or user $k$ in MG $v$ ) can be obtained as

$$
y_{\ell, i, t}^{\mathrm{x}}=\mathbf{h}_{\ell, i, t}^{\mathrm{xH}} \mathbf{w}_{\ell, i, t}^{v} s_{t}^{v}+z_{\ell, i, t}^{\mathrm{x}}, \quad t \in \mathcal{S}_{(\ell, i)},
$$

where $\mathbf{h}_{\ell, i, t}^{\mathrm{x}} \in \mathbb{C}^{N_{\mathrm{T}} \times 1}$ is the channel vector between the AP and user $\mathrm{X}$ over tone $t$ of $R U(\ell, i)$ and $z_{\ell, i, t}^{\mathrm{X}}$ stands for the noise at user $\mathrm{X}$ over that tone. The SNR of user $\mathrm{X}$ over tone $t$ of $R U(\ell, i)$ can be written as

$$
\gamma_{\ell, i, t}^{\mathrm{x}}=\frac{\left|\mathbf{h}_{\ell, i, t}^{\times H} \mathbf{w}_{\ell, i, t}^{v}\right|^{2}}{\sigma_{\ell, i, t}^{\mathrm{x}}}, \quad t \in \mathcal{S}_{(\ell, i)},
$$

where $\sigma_{\ell, i, t}^{\times 2}$ represents the noise power on $R U(\ell, i)$.

1) Effective Signal-to-Noise Ratio: As mentioned above, the data bits in one block are encoded, split, modulated, and transmitted over all tones of a specific RU. Hence, to estimate the BLER corresponding to the MIMO-OFDM transmission over a specific RU, one requires to define a single SNR value that can represent the performance of all tones in that RU. In [22], the exponential effective SNR mapping (EESM) method was proposed to transform multiple SNR values over multiple tones to a so-called effective SNR value. Employing the EESM method, the effective SNR of user $x$ can be obtained as

$$
\gamma_{\ell, i}^{\mathrm{x}}=I^{-1}\left(\frac{1}{S_{(\ell, i)}} \sum_{t \in \mathcal{T}_{(\ell, i)}} I\left(\gamma_{\ell, i, t}^{\mathrm{x}}\right)\right),
$$

where $I(z)$ is defined according to the EESM method as

$$
I(z)=\exp (-z / \zeta(m))
$$

in which $\zeta(m)$ is a setting parameter related to MCS index $m 2$

Remark 1. Regarding the BLER of the OFDM schemes over practical channels as a function of the effective SNR, numerical results given in [25] have demonstrated that at the same value of the effective $S N R$ and the average SNR, the BLER of the data transmission over the practical channels is similar to that obtained over the AWGN channels [23], [24]. Thus, the effective SNR can be considered at the average $S N R$ of the OFDM transmission over an RU. In addition, the BLER in our scheme can be estimated using function given in (4) with respect to the effective SNR.

\section{Joint Link-Adaptation and Resource Allocation Problem}

In this paper, we are interested in jointly optimizing the channelization, RU assignment, precoding vector design, and MCS selection for all UUs/MGs in 802.11ax-based WLAN supporting coexisting unicast and multicast users to maximize the network throughput under a constraint on the AP transmit power budget. In this design, the throughput corresponding to an MG is defined as the sum of the throughputs achieved by all the users in the group. Based on that, the JRRMLA problem can be posed as

$$
\begin{array}{cc}
\max _{\mathbf{A}, \mathbf{W}, \mathbf{m}} & \sum_{\forall(\ell, i)}\left[\sum_{u \in \mathcal{U}^{\cup U}} a_{\ell, i}^{u} T_{\ell, i}^{u}\left(m^{u}, \gamma_{\ell, i}^{u}\right)\right. \\
& \left.+\sum_{n \in \mathcal{G}^{\mathrm{MG}}} a_{\ell, i}^{n} \sum_{k \in \mathcal{U}_{n}^{\mathrm{mul}}} T_{\ell, i}^{n, k}\left(m^{n}, \gamma_{\ell, i}^{n, k}\right)\right] \\
\text { s.t. } \quad & \sum \sum \sum_{t(\ell, i)}\left|\mathbf{w}_{\ell, i, t}^{v}\right|^{2} \leq P_{\mathrm{T}}, \\
& \sum_{\forall(\ell, i)} \sum_{v \in \mathcal{G} \mathcal{U} \mathcal{U}} a_{\ell, i}^{v} \mathbf{b}_{\ell, i} \leq \mathbf{1}^{1 \times 37}, \\
& \sum_{\forall(\ell, i)} a_{\ell, i}^{v}=1, \quad \sum_{v \in \mathcal{G} \mathcal{U}} a_{\ell, i}^{v} \leq 1 \quad \forall v \text { and } \forall(\ell, i), \\
& a_{\ell, i}^{v} \in\{0,1\}, \forall v \text { and }(\ell, i),
\end{array}
$$

where $\mathbf{A}, \mathbf{W}$, and $\mathbf{m}$ stand for all binary RU assignment variables, precoding vectors, and MCS index selection, respectively. The constraints in $10 \mathrm{~d}$ are considered to guarantee a strict RU assignment policy standardized in 802.11ax indicating that each UU/MG is allowed to access only one RU in OFDMA transmission. As can be seen, the optimization problem (10) contains complex variables corresponding to the precoding vector design and integer variables due to the RU assignment and MCS selection. Hence, it is a MINLP which is well-known as an NP-hard problem. In what follows, an efficient solution approach dealing with this challenging problem is discussed.

\footnotetext{
${ }^{2} \zeta(m)$ is estimated based on the BLER curves obtained by simulation results like slope parameters in 44] [22], [23].
} 


\section{Two-Stage Solution ApProach}

The main challenge for solving problem (10) comes from the coupling between the binary RU assignment variables $\mathbf{A}$, integer variables $\mathbf{m}$, and the precoding design $\mathbf{W}$ in the power constraint. In addition, $\mathbf{W}$ consists of $\left(N^{\mathrm{MG}}+N^{\mathrm{UU}}\right) \times N_{\mathrm{T}}$ complex variables that also increase the complexity of solving this problem. Hereby, it is worth recalling that the main issue of beamforming design is to optimize the direction of the precoding vector that adapts to all channel vectors corresponding to all users in MG (or only one UU). Hence, to ease this challenge, a novel two-stage solution approach for solving the JRRMLA problem is presented in this section. In stage one, we aims to determine the temporary precoding vectors for every UU and MG over every RU with a fixed transmission power of $1 \mathrm{~W}$. When the transmission power is updated, the actual precoders can be estimated by up/down scaling the temporary precoding vectors using the square root of the allocated power values. In stage two, new power allocation variables corresponding to the temporary precoders are introduced. Regarding the new variables, the problem stated in (10) can be re-formulated into a joint power allocation, RU assignment, and LA problem. To deal with this relaxed problem, two efficient solution approaches are developed in Section IV Once the problem in phase two is solved, the precoding vectors can be obtained by integrating the temporary solutions achieved in phase one and the power allocation values optimized in phase two.

\section{A. Stage One: Temporary Precoder Design}

This section focuses on designing the temporary precoding vectors corresponding to UUs and MGs over every RU with a fixed transmission power of $1 \mathrm{~W}$. It is worth noting that our objective is to maximize throughput which is calculated based on the exponential BLER functions and the complicated effective SNRs. This objective function is different to that given in the classic beamforming mechanisms studied in literature which mainly focus on maximizing the sum rate or minimum SNR. This requires novel beamforming design approaches which are given in what follows.

1) Temporary Precoder Design for Unicast Transmission: Assume $R U(\ell, i)$ is allocated to UU $u$ for the unicast transmission. For a given MCS index $m^{u}$, the precoder design problem for $\mathrm{UU} u\left(u \in \mathcal{U}^{\mathrm{UU}}\right)$ over $R U(\ell, i)$ with the $1 \mathrm{~W}$ transmission power limit can be formulated as

$$
\begin{aligned}
\min _{\left\{\mathbf{w}_{\ell, i, t}^{u}\right\}^{\prime} s} & \left.e^{-\alpha\left(m^{u}\right) I_{u}^{-1} \mid} \sum_{t \in \mathcal{S}_{(\ell, i)}} I_{u}\left(\left|\mathbf{h}_{\ell, i, t}^{u H} \mathbf{w}_{\ell, i, t}^{u}\right|^{2} / \sigma_{\ell, i, t}^{u 2}\right) / S_{(\ell, i)}\right) \\
\text { s.t. } & \sum_{t \in \mathcal{S}_{(\ell, i)}}\left|\mathbf{w}_{\ell, i, t}^{u}\right|^{2} \leq 1,
\end{aligned}
$$

where $I_{u}(z)=\exp \left(-z / \zeta\left(m^{u}\right)\right)$ and $I_{u}^{-1}(z)=-\zeta\left(m^{u}\right) \ln (z)$. It is worth noting that $\zeta\left(m^{u}\right)$ can be selected as $1 / \alpha\left(m^{u}\right)$ (i.e., $\zeta\left(m^{u}\right)$ is chosen to guarantee the least difference between the estimated BLER and the actual BLER [23]). In this context, problem (11) is equivalent to

$$
\min _{\left\{\mathbf{w}_{\ell, i, t}^{u}\right\}^{\prime}} \sum_{s \in \mathcal{S}_{(\ell, i)}} \exp \left(-\left|\mathbf{h}_{\ell, i, t}^{u H} \mathbf{w}_{\ell, i, t}^{u}\right|^{2} / \sigma_{\ell, i, t}^{u 2}\right) \text { s.t. 11b). }
$$

The optimal solution of this problem is summarized in the following proposition.

Proposition 1. The optimal solution of problem (12) can be expressed as

$$
\begin{aligned}
\mathbf{w}_{\ell, i, t}^{u, \text { temp }} & =\sqrt{p_{\ell, i, t}^{u, \prime}} \mathbf{h}_{\ell, i, t}^{u}, \\
p_{\ell, i, t}^{u, \prime} & =-\frac{1}{\bar{\gamma}_{\ell, i, t}^{u}} \ln \left(\mu\left|\mathbf{h}_{\ell, i, t}^{u H}\right|^{2} / \bar{\gamma}_{\ell, i, t}^{u}\right),
\end{aligned}
$$

where $\bar{\gamma}_{\ell, i, t}^{u}=\left|\mathbf{h}_{\ell, i, t}^{u H}\right|^{4} / \sigma_{\ell, i, t}^{u 2}$ and $\mu$ is a constant so that $\sum_{t \in \mathcal{S}_{(\ell, i)}}\left|\mathbf{h}_{\ell, i, t}^{u}\right|^{2} p_{\ell, i, t}^{u, \prime}=1$.

Proof. The proof is given in Appendix A

2) Temporary Precoder Design for Multicast Transmission: Considering multicast transmission of MG $n$ over $R U(\ell, i)$, the precoder design problem under the $1 \mathrm{~W}$ power-transmission constraint for a given MCS index $m^{n}$ can be represented by

$$
\begin{aligned}
\min _{\left\{\mathbf{w}_{\ell, i, t}^{n}\right\}^{\prime}} & \left.\sum_{s_{k \in \mathcal{U}_{n}^{\text {mul }}}} e^{-\alpha\left(m^{n}\right) I_{n}^{-1}(} \sum_{t \in S_{(\ell, i)}} I_{n}\left(\left|\mathbf{h}_{\ell, i, t}^{n, k, H} \mathbf{w}_{\ell, i, t}^{n}\right|^{2} / \sigma_{\ell, i, t}^{n, k 2}\right) / S_{(\ell, i)}\right) \\
\text { s.t. } & \sum_{t \in \mathcal{S}_{(\ell, i)}}\left|\mathbf{w}_{\ell, i, t}^{n}\right|^{2} \leq 1
\end{aligned}
$$

where $I_{n}(z)=\exp \left(-z / \zeta\left(m^{n}\right)\right)$ and $I_{n}^{-1}(z)=-\zeta\left(m^{n}\right) \ln (z)$. For any MCS index $m^{n}$, by selecting $\zeta\left(m^{n}\right)$ as $1 / \alpha\left(m^{n}\right)$ [23], problem (15) can be re-stated as

$$
\min _{\left\{\mathbf{w}_{\ell, i, t}^{n}\right\}} \sum_{\}^{\prime}} \sum_{k \in \mathcal{U}_{n}^{\text {mul }}} \sum_{t \in \mathcal{S}_{(\ell, i)}} e^{-\left|\mathbf{h}_{\ell, i, t}^{n, k} \mathbf{w}_{\ell, i, t}^{n}\right|^{2} / \sigma_{\ell, i, t}^{2}} \text { s.t. 15b. }
$$

As can be observed, the objective function of the above problem is non-convex and monotonic with respect to the term $\left|\mathbf{h}_{\ell, i, t}^{n, k, H} \mathbf{w}_{\ell, i, t}^{n}\right|^{2}$; hence, problem (16) can be equivalently translated into the following.

$$
\begin{aligned}
& \min _{\mathbf{W}^{n},\left\{g_{\ell, i, t}^{n, k}\right\}} \sum_{k \in \mathcal{U}_{n}^{\text {mul }}} \sum_{t \in \mathcal{S}_{(\ell, i)}} e^{-g_{\ell, i, t}^{n, k}} \\
& \text { s.t. constraint 15b), } \\
& \left|\mathbf{h}_{\ell, i, t}^{n, k, H} \mathbf{w}_{\ell, i, t}^{n}\right|^{2} \geq g_{\ell, i, t}^{n, k} \sigma_{\ell, i, t}^{n, k}{ }^{2}, \quad \forall(k, t),
\end{aligned}
$$

Even after the above transformation, the optimization problem (17) is still non-convex because the constraint $17 \mathrm{~b}$ is in quadratic-greater-than-linear form which is non-convex. To deal with this, we need to approximate it with its lower bound, which can be obtained by a first-order Taylor series approximation. Based on this approximation, an iterative solving approach is proposed to solve the problem (17] [27].

Denote $f\left(\mathbf{w}_{\ell, i, t}^{n}\right)=\mathbf{w}_{\ell, i, t}^{n H} \mathbf{H}_{\ell, i, t}^{n, k} \mathbf{w}_{\ell, i, t}^{n}$ where $\mathbf{H}_{\ell, i, t}^{n, k}=$ $\mathbf{h}_{\ell, i, t}^{n, k} \mathbf{h}_{\ell, i, t}^{n, k, H}$ and $\mathbf{w}_{\ell, i, t}^{n,(s)}$ as the $s^{\text {th }}$ iteration point of $\mathbf{w}_{\ell, i, t}^{n}$. 


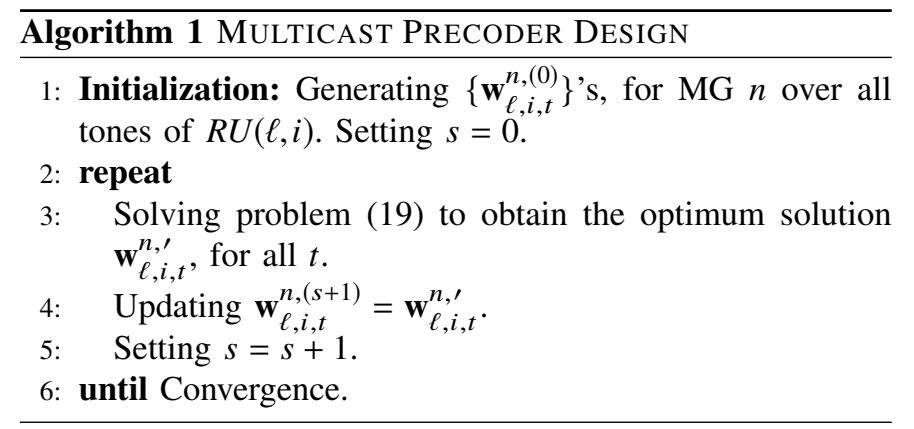

Then, the first-order Taylor series approximation of $f\left(\mathbf{w}_{\ell, i, t}^{n}\right)$ in iteration $s$ can be given as

$$
\bar{f}\left(\mathbf{w}_{\ell, i, t}^{n}, \mathbf{w}_{\ell, i, t}^{n,(s)}\right)=f\left(\mathbf{w}_{\ell, i, t}^{n,(s)}\right)+2 \mathfrak{R}\left\{\mathbf{w}_{\ell, i, t}^{n H} \mathbf{H}_{\ell, i, t}^{n, k} \mathbf{w}_{\ell, i, t}^{n,(s)}\right\},
$$

Eventually, in iteration $s$, problem (17) can be reformulated as

$$
\begin{aligned}
\min _{\mathbf{w}^{n},\left\{g_{\ell, i, t}^{n, k}\right\}} & \sum_{\text {s.t. }} \\
& \sum_{k \in \mathcal{U}_{n}^{\text {mul }}} \sum_{t \in \mathcal{S}_{(\ell, i)}} e^{-g_{\ell, i, t}^{n, k}} \\
& \left|\mathbf{w}_{\ell, i, t}^{n}\right|^{2} \leq 1, \\
& \bar{f}\left(\mathbf{w}_{\ell, i, i}^{n}, \mathbf{w}_{\ell, i, t}^{n,(s)}\right) \geq g_{\ell, i, t}^{n, k} \sigma_{\ell, i, t}^{n, k} \quad \forall(k, t) .
\end{aligned}
$$

This problem is a convex second-order cone programming (SOCP), which can be efficiently solved by convex solvers such as CVX [28]. We summarize the procedure to update $\mathbf{w}_{\ell, i, t}^{n}$ in Algorithm 11 which is employed to solve the problem (16).

Remark 2. It is worth noting that when the EESM is chosen to guarantee the least difference between the estimated BLER and the actual BLER as discussed in [23], i.e., $\zeta(m)$ can be selected as $1 / \alpha(m)$ for every MCS index $m$, the temporary precoder direction achieved in this section for every UU/MG can be kept unchanged for all MCS selections.

\section{B. Stage Two: Joint Power Control, RU Assignment, and LA Problem}

Let $\bar{\gamma}_{\ell, i}^{\mathrm{x}}$ be the effective SNR of user $\mathrm{x}$ (UU $v$ or user $k$ in MG v) which is obtained based on the temporary precoder solution of the problem (12) or (16). Denote $p_{\ell, i}^{v}$ as the power allocation for UU/MG $v(v \in \mathcal{G} \mathcal{U})$ over $R U(\ell, i)$. Then, the effective SNRs of UU $u$ and user $k$ in MG $n$ over $R U(\ell, i)$ can be estimated as

$$
\begin{aligned}
\gamma_{\ell, i}^{u} & =\bar{\gamma}_{\ell, i}^{u} p_{\ell, i}^{u}, \forall v \in \mathcal{U}^{\mathrm{UU}} \\
\gamma_{\ell, i}^{n, k} & =\bar{\gamma}_{\ell, i}^{n, k} p_{\ell, i}^{n}, \forall k \in \mathcal{U}_{n}^{\mathrm{mul}}, n \in \mathcal{G}^{\mathrm{MG}}
\end{aligned}
$$

The stage two's goal is to determine the RUs allocation, transmission power, and MCS selection for all UUs/MGs. Using [20)-21], problem (10) can be re-stated as follows,

$$
\begin{array}{r}
\max _{\mathbf{A}, \mathbf{p}, \mathbf{m}} \sum_{\forall(\ell, i)}\left[\sum_{n \in \mathcal{G}^{\mathrm{MG}}} a_{\ell, i}^{n} \sum_{k \in \mathcal{U}_{n}^{\mathrm{mul}}} T_{\ell, i}^{n, k}\left(m^{n}, \bar{\gamma}_{\ell, i}^{n, k} p_{\ell, i}^{n}\right)\right. \\
\left.+\sum_{u \in \mathcal{U}^{\mathrm{UU}}} a_{\ell, i}^{u} T_{\ell, i}^{u}\left(m^{u}, \bar{\gamma}_{\ell, i}^{u} p_{\ell, i}^{u}\right)\right]
\end{array}
$$

$$
\begin{aligned}
& \text { s.t. } \\
& \sum_{\forall(\ell, i)} \sum_{v \in \mathcal{G} \mathcal{U}} p_{\ell, i}^{v} \leq P_{\mathrm{T}},
\end{aligned}
$$

where $\mathbf{p}$ stands for all power allocation variables. This problem is an MINLP which can be solved by using some solver tools such as GAMS, CPLEX, MINLP, etc. [29]. However, this problem is well-known as NP-hard and therefore requires exponential-complexity computing time to be solved. Hence, some efficient low-complexity solution approaches dealing with this problem are still required in real-time and practical systems. Let $\mathbf{p}^{\star}, \mathbf{a}^{\star}$, and $\mathbf{m}^{\star}$ be the optimum solution of problem (22). Then, $\mathbf{a}^{\star}$, and $\mathbf{m}^{\star}$ will also be the RU assignment and MCS selection solutions of the JRRMLA problem while the beamforming solution corresponding to user $\mathrm{X}$ over tone $t$ of $R U(\ell, i)$ can be estimated as

$$
\mathbf{w}_{\ell, i, t}^{\mathrm{x}, \star}=\sqrt{p_{\ell, i}^{\mathrm{x}, \star}} \mathbf{w}_{\ell, i, t}^{\mathrm{x}, \mathrm{temp}}
$$

where $\mathbf{w}_{\ell, i, t}^{\mathrm{x} \text {,emp }}$ represents the temporary precoder of user $\mathrm{x}$ over tone $t$ of $R U(\ell, i)$ obtained in stage one.

\section{Low-Complexity Joint Power Allocation, RESOURCE UNIT ASSIGNMENT, AND LINK ADAPTATION ALGORITHMS}

This section presents two low-complexity algorithms to deal with problem (22). The first is developed based on the wellknown compressed sensing method while the second is to optimize each of the optimizing variables alternatively until reaching converged outcomes. The details are given in what follows.

\section{A. Compressed Sensing-based Algorithm}

The main challenges in solving (22) come from the coupling of binary variables $\left\{a_{\ell, i}^{v}\right\}$ and integer variable $\mathbf{m}$, and the nonlinear form of the objective function. However, there is no coupling between $\mathbf{m}$ and other variables in the constraints. Hence, once $\mathbf{A}$ and $\mathbf{p}$ are determined, $\mathbf{m}$ can be optimized to maximize the throughput of every UU and MG. We can alternatively solve 22) for a given $\mathbf{m}$; then update $\mathbf{m}$ after $\mathbf{A}$ and $\mathbf{p}$ are optimized. Regarding this approach, we first re-state problem 22) for a given $\mathbf{m}$ as

$$
\begin{aligned}
& \min _{\mathbf{A}, \mathbf{p}} \sum_{\forall(\ell, i)}\left[\sum_{n \in \mathcal{G}^{\mathrm{MG}}} a_{\ell, i}^{n} \omega^{n} \sum_{k \in \mathcal{U}_{n}^{\mathrm{mul}}} e^{-\alpha_{\ell, i}^{n, k} p_{\ell, i}^{n}}\right. \\
&\left.+\sum_{u \in \mathcal{U}^{\mathrm{UU}}} a_{\ell, i}^{u} \omega^{u} e^{-\alpha_{\ell, i}^{u} p_{\ell, i}^{u}}\right]
\end{aligned}
$$

s.t. constraints 10c, 10d, 10e, 22b,

where $\omega_{\ell, i}^{v}=\theta_{\ell, i}^{v}\left(m^{v}\right) A\left(m^{v}\right)(\forall v \in \mathcal{G U})$ and $\alpha_{\ell, i}^{\mathrm{x}}=\alpha\left(m^{v}\right) \bar{\gamma}_{\ell, i}^{\mathrm{x}}$ (x stands for UU $v$ or user $k$ in MG $v$ ). As can be observed, $p_{\ell, i}^{v}=0$ implies that $R U(\ell, i)$ is not assigned to UU/MG $v$, i.e., $a_{\ell, i}^{v}=0$. Inversely, if $p_{\ell, i}^{v}>0$ then $a_{\ell, i}^{v}$ should be 1 . Hence, we can indicate that

$$
a_{\ell, i}^{v}=\left\|p_{\ell, i}^{v}\right\|_{0}, \forall v,(\ell, i)
$$


where $\|.\|_{0}$ is the $\ell_{0}$-norm. Based on this observation, the binary variables $\left\{a_{\ell, i}^{v}\right\}$ can be omitted by employing the $\ell_{0^{-}}$ norm of the power variables. However, the problem is still very challenging due to the $\ell_{0}$-norm terms in the constraint. To overcome this point, one can employ an iterative CSbased algorithm which consists of the following process in each $s+1^{\text {th }}$ iteration: (i) replacing the sparsity term $\|x\|_{0}$ by $\left(1 /\left|x^{[s]}\right|^{2}+\varepsilon\right)^{1 / 2} x$ where $\varepsilon$ is sufficiently small; (ii) solving the approximate problem; (iii) updating the fixed point for the next iteration based on the recent achieved optimal solution. The efficiency of employing this CS-based approach to solve a sparsity problem has been confirmed in many works [30][32]. Based on this approach, in the $s+1^{t h}$ iteration, problem (24) can be approximated as

$$
\begin{aligned}
\min _{\mathbf{p}} \sum_{\forall(\ell, i)}\left[\sum_{n \in \mathcal{G}^{\mathrm{MG}}} \omega_{\ell, i}^{n} \sum_{k \in \mathcal{U}_{n}^{\mathrm{mul}}} e^{-\alpha_{\ell, i}^{n, k} p_{\ell, i}^{n}}\right. & \\
& \left.+\sum_{u \in \mathcal{U}^{\mathrm{UU}}} \omega_{\ell, i}^{u} e^{-\alpha_{\ell, i}^{u} p_{\ell, i}^{u}}\right]
\end{aligned}
$$

s.t. constraint 22b),

$$
\begin{aligned}
& \sum_{\forall(\ell, i)} \sum_{v \in \mathcal{G} \mathcal{U}} \delta_{\ell, i}^{v} p_{\ell, i}^{v} \mathbf{b}_{\ell, i} \leq \mathbf{1}^{1 \times 37}, \\
& \sum_{\forall(\ell, i)} \delta_{\ell, i}^{v} p_{\ell, i}^{v}=1, \quad \forall v \in \mathcal{G} \mathcal{U} \\
& \sum_{v \in \mathcal{G} \mathcal{U}} \delta_{\ell, i}^{v} p_{\ell, i}^{v} \leq 1, \forall(\ell, i),
\end{aligned}
$$

where the weight $\delta_{\ell, i}^{v}$ is expressed as

$$
\delta_{\ell, i}^{v}=\left(p_{\ell, i}^{v[s]^{2}}+\varepsilon\right)^{-1 / 2}, \forall v \in \mathcal{G} \mathcal{U}
$$

This problem is convex due to the convex objective function and the linear-form constraints; hence, it can be solved efficiently by employing convex solvers such as CVX [28] or other benchmark searching methods, such as gradient descent. The convergence of this iterative approach has been established in many works related to CS [30]-[32]. In addition, the outcome is a good solution satisfying all the initial sparsity constraints. After obtaining the solution $\mathbf{p}^{\star}, p_{\ell, i}^{v}$ with very small value, i.e. less than $\varepsilon$, will be rounded to zero. Then, the RU assignment can be determined by defining $a_{\ell, i}^{v}$ as in (25), and MCS indices will be selected to maximize the throughput. Finally, the solution approach for solving problem [10) is summarized in Algorithm 2 .

\section{B. Alternative Radio Resource Management Algorithm}

In this section, another iterative solution method, namely "Alternative Radio Resource Management Algorithm" is proposed. The idea behind this approach is that one of the design variables corresponding to the power allocation, MCS selection and RU assignment can be optimized while the others are kept unchanged. In particular, the power allocation can be solved optimally through the water-filling approach when A and $\mathbf{m}$ are fixed [38]. The MCS selection $\mathbf{m}$ corresponding to the transmission over a specific RU can be optimized

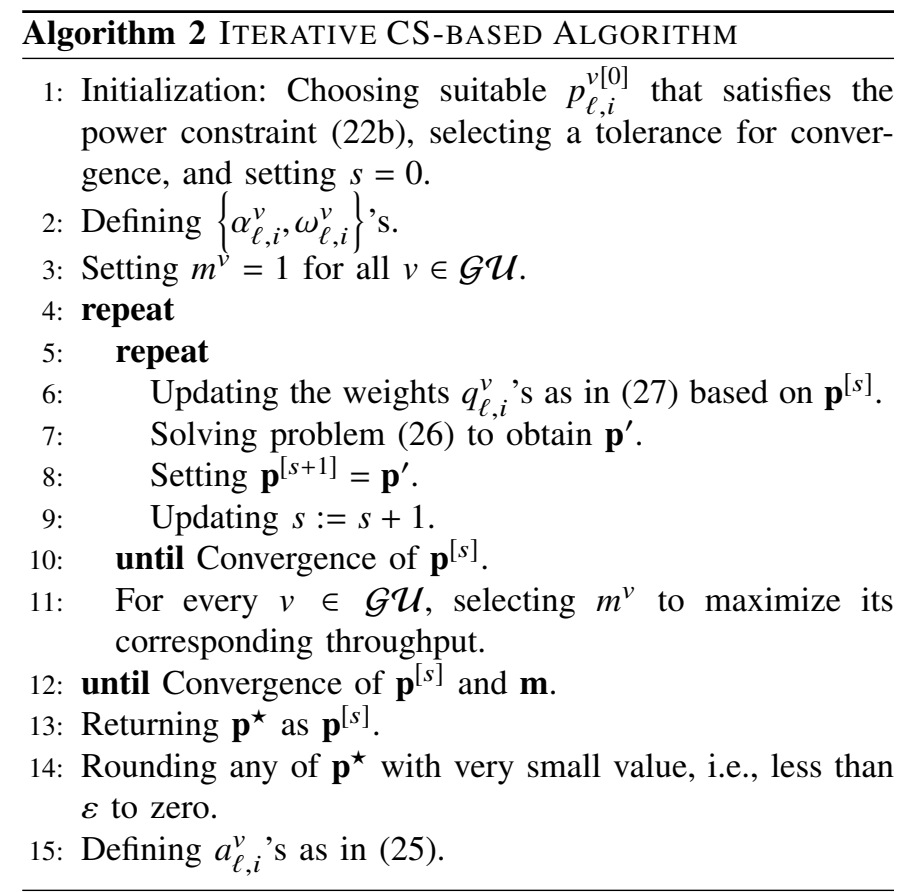

easily once $\mathbf{p}$ and $\mathbf{A}$ are known. Then, when $\mathbf{m}$ and $\mathbf{p}$ are given, the RU assignment can be solved efficiently by transferring the remaining design problem into a traditional Assignment Problem with Constraints (APC). In this problem, the weight of each one-to-one matching between RUs and UUs/MGs is estimated as the corresponding throughput value. The APC problem can be solved efficiently by employing several methods given in [33], [34]. Assuming p, m, and A are being optimized in each iteration, this iterative solution approach is described in details in what follows.

1) Power Allocation Optimization for given RU Assignment and MCS Selection: Here, we assume that $\mathbf{A}$ and $\mathbf{m}$ are fixed and set to the value obtained from the previous iteration. Then, we optimize the power allocation as follows. To achieve the optimum power solution, we re-cast the problem in 22 for a given $\mathbf{A}$ and $\mathbf{m}$ as

$$
\begin{aligned}
\min _{\mathbf{p}} \sum_{\forall(\ell, i)}\left[\sum_{n \in \mathcal{G}^{\mathrm{MG}}} a_{\ell, i}^{n} \omega_{\ell, i}^{n} \sum_{k \in \mathcal{U}_{n}^{\mathrm{mul}}} e^{-\alpha_{\ell, i}^{n, k} p_{\ell, i}^{n}}\right. \\
\left.+\sum_{u \in \mathcal{U}^{\mathrm{UU}}} a_{\ell, i}^{u} \omega_{\ell, i}^{u} e^{-\alpha_{\ell, i}^{u} p_{\ell, i}^{u}}\right]
\end{aligned}
$$

s.t. constraint 22b.

As can be seen, this is a convex problem due to the exponential form of the objective function and the power constraint. Then, its optimal solution can be determined according to the following proposition.

Proposition 2. The optimal solution of problem (28) can be expressed as

- If $a_{\ell, i}^{v}=0$, then $p_{\ell, i}^{v *}=0$.

- If $a_{\ell, i}^{v, i}=1$, then we have

$$
p_{\ell, i}^{u *}=\frac{1}{\alpha_{\ell, i}^{u}} \ln \left(\omega_{\ell, i}^{u} \alpha_{\ell, i}^{u} / \rho\right), \forall u \in \mathcal{U}^{\mathrm{UU}}
$$




$$
\begin{aligned}
& \text { and } p_{\ell, i}^{n *}=\left.p^{\prime}\right|_{f_{\ell, i}^{n}\left(p^{\prime}\right)=\rho}, \forall n \in \mathcal{G}^{\mathrm{MG}}, \\
& \text { where } f_{\ell, i}^{n}(p)=\omega_{\ell, i}^{n} \sum_{k \in \mathcal{U}_{n}^{\text {mul }}} \alpha_{\ell, i}^{n, k} \exp \left(-\alpha_{\ell, i}^{n, k} p\right) \text {, and } \rho \text { is } \\
& \text { a constant so that } \sum_{v \in \mathcal{G} \mathcal{U} \forall(\ell, i)} p_{\ell, i}^{v *}=P_{\mathrm{T}} \text {. }
\end{aligned}
$$

Proof. The proof is given in Appendix B

It is worth noting that $\mathbf{p}^{*}$ solution in each iteration given in Proposition 2 is in sparse form; specifically, there is only one $p_{\ell, i}^{v}$ that is greater than 0 for UU/MG $v$ over all $R U(\ell, i)$ while the others are zeros.

2) MCS Selection Optimization for Given Power Transmission and RU Assignment: Once the optimal power allocation $\mathbf{p}^{*}$ is determined according to Proposition 2, one can optimize the MCS indices to maximize the throughputs corresponding to the UUs and MGs as follows,

$$
\begin{aligned}
& m^{u *}=\arg \max _{m} \sum_{\forall(\ell, i)} a_{\ell, i}^{u} T_{\ell, i}^{u}\left(m, \bar{\gamma}_{\ell, i}^{u} p_{\ell, i}^{u *}\right), \forall u \in \mathcal{U}^{\mathrm{UU}}, \\
& m^{n *}=\arg \max _{m} \sum_{\forall(\ell, i)} a_{\ell, i}^{n} \sum_{k \in \mathcal{U}_{n}^{\text {mul }}} T_{\ell, i}^{n, k}\left(m, \bar{\gamma}_{\ell, i}^{n, k} p_{\ell, i}^{n *}\right), \forall n \in \text { (GM) }
\end{aligned}
$$

3) $R U$ Assignment Designs for Given Power Transmission and MCS Selection: For a given $\mathbf{p}^{*}$ and $\mathbf{m}^{*}$ which are achieved in each iteration or assigned in the initialization stage, the RU assignment will be updated as follows. Apart from the initialization stage, the power allocation for UU/MG $v$ is optimized according to Proposition 2 based on the solution of $\mathbf{A}$ from the previous iteration; hence, it is in a sparse form. If the weights corresponding to all one-to-one matching links between RUs and UUs/MGs are calculated as the throughput based on $\mathbf{p}^{*}$, the weights are also in a sparse form. This makes the RU-UU/MG assignment, $\mathbf{A}$, remaining unchanged in this iteration. Then, the optimization process is trapped in an inefficient solution. To overcome this challenge, let $p^{v}$ be a new value representing the transmission power allocated to UU/MG $v$ in this iteration, which can be obtained as

$$
p^{v}=\sum_{\forall(\ell, i)} p_{\ell, i}^{v *}
$$

Then, the weight corresponding to the one-to-one RU and UU/MG matching between $R U(\ell, i)$ and UU/MG $v$ can be estimated as

$$
\begin{aligned}
& q_{\ell, i}^{u}=T_{\ell, i}^{u}\left(m^{u}, \bar{\gamma}_{\ell, i}^{u} p^{u}\right), \forall(\ell, i) \text { if } u \in \mathcal{U}^{\mathrm{UU}}, \\
& q_{\ell, i}^{n}=\sum_{k \in \mathcal{U}_{n}^{\mathrm{mul}}} T_{\ell, i}^{n, k}\left(m^{n}, \bar{\gamma}_{\ell, i}^{n, k} p^{n}\right), \forall(\ell, i) \text { if } n \in \mathcal{G}^{\mathrm{MG}} .
\end{aligned}
$$

Based on the estimated weights, the RU assignment problem is rewritten as

$$
\max _{\mathbf{A}} \sum_{\forall(\ell, i)} \sum_{v \in \mathcal{G} \mathcal{U}} q_{\ell, i}^{v} a_{\ell, i}^{v} \text { s.t. 110c, 10d, 10e. }
$$

This problem is an APC problem - a type of Integer Linear Programming (ILP), which can be solved optimally by some well-known algorithms [41] or optimization solvers [28], [29]. This solution approach can be summarized in Algorithm 3 in which the $p^{v}$ 's are set the same at the initialization, i.e., $p^{v}=P_{\mathrm{T}} /\left(N^{\mathrm{UU}}+N^{\mathrm{MG}}\right)$, and $m^{v[0]}=1$. Then, in each iteration,

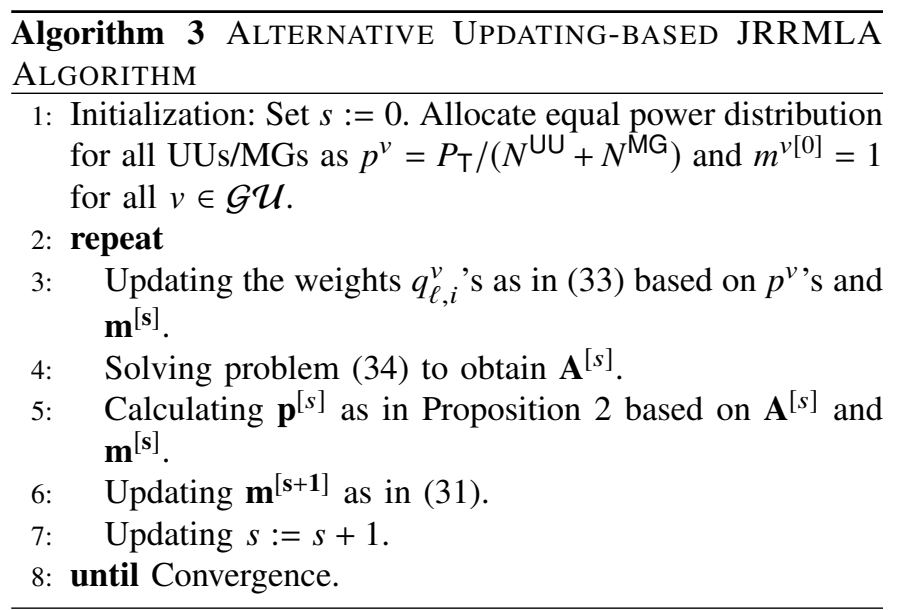

$\mathbf{A}, \mathbf{p}$, and $\mathbf{m}$ are alternatively optimized. The convergence of this algorithm is discussed in the following proposition.

Proposition 3. Algorithm 3 converges after a finite number
of iterations.

Proof. Since the problems (34), (28), and the procedure of optimizing $\mathbf{m}$ as given in equation (31) aim to maximize the total throughput, the objective function of problem (22) is monotone and increases in each iteration. Due to the fact that it cannot increase to infinity, the convergence of Algorithm 3 can be guaranteed.

\section{COMPlexity AnAlysis And Other Solution APPROACH}

\section{A. Complexity Analysis}

Our design approach includes two stages. The first is for the temporary precoder design for every UU/MG over each RU which is presented in Section III-A. While the second stage focuses on the solution frameworks for power allocation, RU assignment, and MCS selection which can be carried by Algorithms 2 and 3 in Section IV This section analyses the complexity of our designs based on the number of required computations. Note that the transmission over $R U(0,0)$ with biggest number of tones $(S=996)$ is considered to estimate the highest order of computation.

1) Stage-One: Regarding Proposition 1 , the temporary pre-

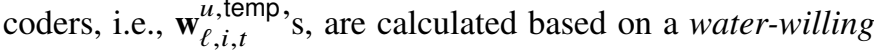
algorithm in which a water level $\mu$ and power transmission over every tone are updated iteratively until the power constraint is satisfied. As reported in [38], the number of iterations of the general water-filling algorithm grows linearly with the number of variables. Moreover, $S$ transmission power values need to be updated according to the new level in each iteration. Therefore, the complexity of the unicast precoder design can be estimated as

$$
X_{\mathrm{UU}-\mathrm{BF}}=O\left(S^{2}\right),
$$

For the multicast precoder design given in Algorithm 1, one requires to solve the SOCP problem in (19) in each iteration. The worst-case complexity of solving an SOCP is reported in Chapter 6 of $[39]$ as $O\left(\sqrt{Y} z^{3}\right)$, where $z$ is the number of 
variables and $Y$ is the number of second-order cone constraints. Since, problem (19) corresponding to $\mathrm{MG} n$ has $U_{n}^{\mathrm{mul}} S\left(N_{\mathrm{T}}+1\right)$ variables and $U_{n}^{\mathrm{mul}} S+1$ constraints, the complexity of multicast precoder design can be given as

$$
X_{\mathrm{MG}-\mathrm{BF}}=O\left(I_{\mathrm{alg} 1} N_{\mathrm{T}}\left(U_{n}^{\mathrm{mul}} S\right)^{3.5}\right),
$$

where $I_{\mathrm{alg} 1}$ stands for the number of iterations of Algorithm 1 for obtaining the precoding vectors.

2) Stage-Two: We now quantify the complexity of solving problem (22) in stage two, i.e., using Algorithms 2 or 3 In Algorithm 2, each iteration tries to solve problem (26). This is a convex problem which can be solved by employing the standard gradient descent method [40]. Typically, the complexity of the gradient descent algorithm is in the order of $O\left(z \zeta_{2}^{-2}\right)$, where $z$ is the number of variables and $\zeta_{s}$ is the solution accuracy [40]. Hence, the complexity of Algorithm 2 can be given as

$$
X_{\mathrm{alg} 2}=O\left(I_{\mathrm{alg} 2} M_{\mathrm{RU}} N \zeta_{2}^{-2}\right),
$$

where $N=N^{\mathrm{UU}}+N^{\mathrm{MG}}$ stands for the total number of UUs and MGs, $M_{\mathrm{RU}}$ is the total number of RUs, and $I_{\mathrm{alg} 2}$ represents the number of iterations for solving problem (22) using Algorithm 2 .

In Algorithm 3, in each iteration, $p_{\ell, i}^{v}, m^{v}$, and the RUUU/MG assignment are updated. The complexity of power updating is $O\left(N^{2}\right)$ due to the water-filling approach [38], while the computation effort for updating $m^{v}$ is of $12 N$ as shown in 31. To estimate the computation complexity associated to solving the APC problem in (34), one needs to regard this problem as a graph matching problem with $n_{\mathrm{V}}=N+M_{\mathrm{RU}}$ nodes and $m_{\mathrm{e}}=N M_{\mathrm{RU}}$ edges [41]. This problem can be solved efficiently using the "Generic Cost Scaling Algorithm" presented in [41]. The complexity of this algorithm is in the order of $O\left(n_{\mathrm{v}}^{2} m_{\mathrm{e}} \log \left(n_{\mathrm{v}} C_{\mathrm{SC}}\right)\right)$, where $C_{\mathrm{Sc}}$ is cost scaling factor. Since the computation effort for solving problem 34 dominates that of updating $p_{\ell, i}^{v}$ and $m^{v}$, the complexity of Algorithm 3 can be approximated as

$$
X_{\mathrm{alg} 3}=O\left(I_{\mathrm{alg} 3}\left(N+M_{\mathrm{RU}}\right)^{2} N M_{\mathrm{RU}} \log \left(N+M_{\mathrm{RU}} C_{\mathrm{SC}}\right)\right),
$$

where $I_{\mathrm{alg} 3}$ represents the number of iterations for solving problem 22, using Algorithm 3

\section{B. Greedy Solution Approach}

For comparison purposes, we also consider a greedy algorithm for solving the optimization problem (10) in this section. This algorithm is developed based on the work given in [14]. In particular, Wang et. al in [14] have proposed a recursive algorithm for WiFi 802.11ax systems which implements two tasks: (i) channelizing the bandwidth into a combination of RUs and (ii) allocating these RUs to users or group of users to maximize the transmission rate. In addition, equal power transmission and Zero-Forcing beamforming are employed in this work. To adapt this solution to our scheme, some modifications are required as detailed bellow.

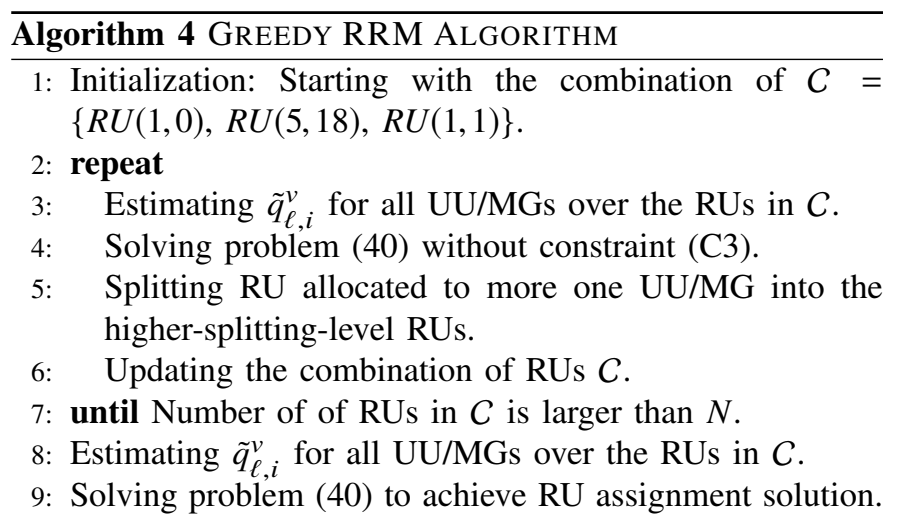

1) Precoding design: For the beamforming design, we adopt the results given in Section III-A with the equal transmission power allocation. Then, the precoding vector of user x (UU or MU) over tone $t$ of $R U(\ell, i)$ can be written as

$$
\tilde{\mathbf{w}}_{\ell, i, t}^{\mathrm{x}}=\sqrt{p_{\text {eq }}} \mathbf{w}_{\ell, i, t}^{\mathrm{x} \text { temp }}
$$

where $p_{\text {eq }}=P_{\mathrm{T}} / N$.

2) Greedy Resource Allocation Solution: The channelization and resource allocation due to this greedy algorithm is designed according to the approach given in [14]. The basic idea behind this solution is to optimize the RU assignment for a given combination of RUs without regarding the strict constraint that one RU is assigned to only one UU/MG. If an RU is assigned to more than one UU/MG, it will be split into the higher-splitting-level RUs. The RU assignment is reoptimized with the new combination of RUs. The process is repeated until the number of RUs is larger than the total number of UUs/MGs. Then, one-to-one RU-UU/MG matching to maximize the throughput is performed to achieve the final RU assignment. Let $\tilde{q}_{\ell, i}^{v}$ be the throughput of UU/MG $v$ over $R U(\ell, i)$ achieved based on precoders given in (39) and MCSs optimized as in 31. Then, the one-to-one RU-UU/MG matching problem is given as

$$
\begin{array}{ll}
\max _{\mathbf{A}} & \sum_{R U(\ell, i) \in C} \sum_{v \in \mathcal{G} \mathcal{U}} \tilde{q}_{\ell, i}^{v} a_{\ell, i}^{v} \\
\text { s.t. } & (C 1): a_{\ell, i}^{v} \in\{0,1\}, \forall(v, \ell, i), \\
& (C 2): \sum_{\forall(\ell, i)} a_{\ell, i}^{v}=1 \forall v, \\
& (C 3): \sum_{v \in \mathcal{G} \mathcal{U}} a_{\ell, i}^{v} \leq 1 \quad \forall(\ell, i) .
\end{array}
$$

Here, the RU assignment without the constraint of that one RU is assigned to only one UU/MG can be presented as problem (40) without constraint (C3). This assignment problem is simpler than the APC problem given in (34) and can be solved by employing the well-known Hungarian method (i.e., Algorithm 14.2.3 given in [45]). Assuming $N>1$, the process is summarized in Algorithm 4 . In the following section, this algorithm is referred to "Wang's Alg." and its numerical results will be presented for comparison purposes. 
TABLE III

SLOPE PARAMETERS AND DROPPING POINT FOR BLER APPROXIMATION WITH DIFFERENT MCS INDICES IN WIFI 802.11 AX.

\begin{tabular}{|l|l|l|l|l|l|l|l|l|l|l|l|l|}
\hline$m$ & 0 & 1 & 2 & 3 & 4 & 5 & 6 & 7 & 8 & 9 & 10 & 11 \\
\hline$\alpha(m)$ & $5.15 e-2$ & $2.225 e-2$ & $1.17 e-2$ & $4.15 e-3$ & $1.78 e-3$ & $9.39 e-4$ & $8.18 e-4$ & $5.07 e-4$ & $1.94 e-4$ & $1.98 e-4$ & $5.83 e-5$ & $4.34 e-5$ \\
\hline$\beta(m)$ & 2.04 & 6.06 & 14.27 & 21.2 & 61.16 & 85.59 & 105.73 & 203.88 & 412.77 & 911.23 & 1258.1 & 1880.8 \\
\hline
\end{tabular}

\section{NUMERICAL RESUlTS}

\section{A. Slope Value and Dropping Point Estimation}

The joint beamforming and resource allocation design frameworks have been proposed based on the general BLER function and any values of $\alpha(m)$ and $\beta(m)$. The remaining work is to select the accurate BLER function by determining the right slope parameter and dropping point. i.e., $\alpha(m)$ and $\beta(m)$, for each MCS index. One traditional approach to estimate the parameters of a modelling function is the curve-fitting method that provides the best fit to a specific dataset [46]. The accuracy of the estimated parameters mainly depends on the correctness of the input dataset [46]. However, collecting the precise BLER data at a certain value of effective SNR through Monte-Carlo simulation is challenging since the effective SNR value is unpredictable and unsettled in the fading environment. Thanks to the results given in [23]-[26], Remark 1 suggests us to estimate these parameters based on the BLER accuracy achieved through simulations over the AWGN channels.

Therefore, this section aims to estimate $\alpha(m)$ and $\beta(m)$ corresponding to all MCS schemes in (4) by estimating the BLER achieved over the AWGN channels in the 802.11axbased WLAN. To do so, the "802.11ax Packet Error Rate Simulation" process obtained from the Matlab WLAN Toolbox [35] is adopted to collect the simulated BLERs of 12 MCS schemes over different values of the average SNR for an 802.11ax-based WLAN system utilizing LPDC encoder and the AWGN channel model. Based on the collected data of BLER, the nonlinear curve-fitting tool is applied to approximate the values of $\alpha(m)$ and $\beta(m)$ in (4) for every MCS. In these simulations, we set $L^{v}=1500$ bytes and $O^{v}=10 \%$ of $L^{v}$ for all $v \in \mathcal{G U}$. The estimated parameters are then summarized in Table III while the BLER over various average SNR values corresponding to the simulated data and fitting tool are illustrated in Fig. 2

\section{B. Simulation Results}

This section presents illustrative numerical results that demonstrate the performance of the proposed algorithms. In all simulations, the AP is placed at the center of the network while all users are randomly distributed inside the ring with minimum and maximum radii, $d_{\min }$ and $d_{\max }$, respectively. An example of the network settings for our simulations are illustrated in Fig 3. The channel vectors from the AP to all users are generated according to the path loss model,

$$
P L_{\text {overall }}=P L_{\text {indoor }}(d)+P E L_{\text {wall }}+P E L_{\text {shadow }},
$$

where $d$ is the distance separating the AP from a specific user, $P L_{\text {indoor }}(d)$ is estimated as $20 \log _{10}(d)$ if $d \leq d_{\mathrm{BP}}$ and $35 \log _{10}(d)-15 \log _{10}\left(d_{\mathrm{BP}}\right)$ if $d>d_{\mathrm{BP}}$, and $P E L_{\text {shadow }}$ stands for the shadow fading loss. Moreover, $P E L_{\mathrm{wall}}=n_{\mathrm{w}} L_{\mathrm{w}}$,

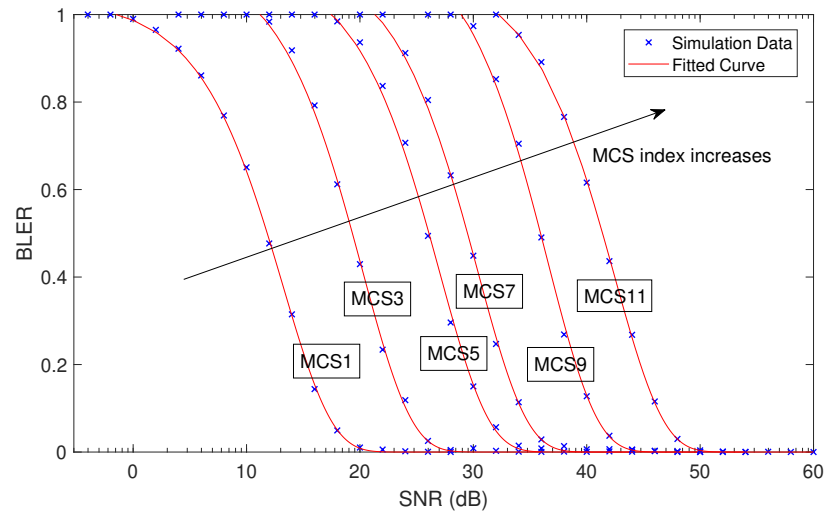

Fig. 2. BLER versus the average SNR achieved by simulation and the curvefitting tool of Matlab.

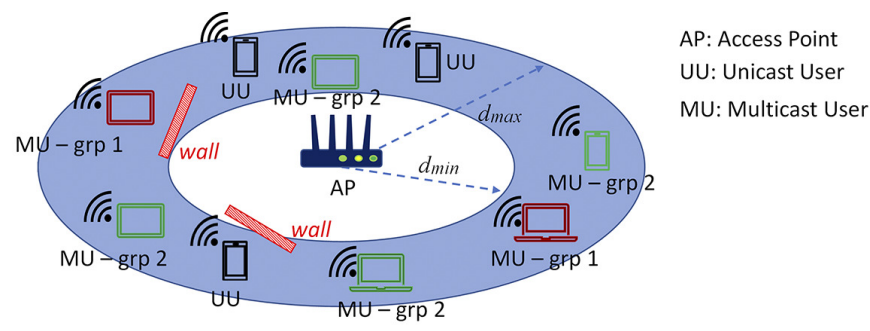

Fig. 3. The simulated WiFi IEEE 802.11ax network setting.

$n_{\mathrm{w}}$ is the number of walls penetrated and $L_{\mathrm{w}}=5(\mathrm{~dB})$ is the penetration loss for a single wall. In our simulations, two indoor environment models from the IEEE Wireless LAN specifications [42] are considered, namely model B for residential indoor scenarios, and model $\mathbf{F}$ for large space indoor (pseudo outdoor) scenarios. The setting parameters corresponding to these models are summarized in Table. IV In addition, the number of walls between the AP and each user are randomly chosen from the set $\{0,1,2\}$.

We first aim to estimate the achievable throughput of 802.11ax-based WLAN within a residential indoor environment where the channel model $\mathbf{B}$ in the Matlab WLAN Toolbox is employed to generate the channel vectors [35], [36]. The simulation results corresponding to this scenario are presented in Figs. 4 8 . In these simulations, there are three MGs which consist of 3, 4, 5 multicast users, respectively, while the number of UUs is set to $4, N^{\mathrm{UU}}=4$, except in the simulation corresponding to Fig 8 Additionally, the minimum and maximum radii of user-location ring are 8 and $12 \mathrm{~m}$, respectively. The noise power spectral density is set to $-174 \mathrm{dBm} / \mathrm{Hz}$ [37]. Moreover, unless indicated otherwise, $P_{\mathrm{T}}=200 \mathrm{~mW}$ and $N_{\mathrm{T}}=4$. 
TABLE IV

PROPAGATION AND PATH LOSS PARAMETERS OF INDOOR WIFI 802.11AX CHANNEL MODELS [42].

\begin{tabular}{|l|l|l|l|l|l|l|l|}
\hline Model & $\begin{array}{l}\text { Delay } \\
\text { spread (ns) }\end{array}$ & $\begin{array}{l}\text { Number of } \\
\text { clusters }\end{array}$ & Taps/Cluster & Propagation Scenario & $d_{\mathrm{BP}}(\mathrm{m})$ & $\begin{array}{l}\text { Shadow fading } \\
\text { before } d_{\mathrm{BP}}(\mathrm{dB})\end{array}$ & $\begin{array}{l}\text { Shadow fading } \\
\text { after } d_{\mathrm{BP}}(\mathrm{dB})\end{array}$ \\
\hline $\mathrm{B}$ & 15 & 2 & 5,7 & Indoor Residential & 5 & 3 & 4 \\
\hline $\mathrm{F}$ & 150 & 6 & $15,12,7,3,2,2$ & $\begin{array}{l}\text { Large Space Indoor } \\
\text { (pseudo-outdoor) }\end{array}$ & 30 & 3 & 6 \\
\hline
\end{tabular}

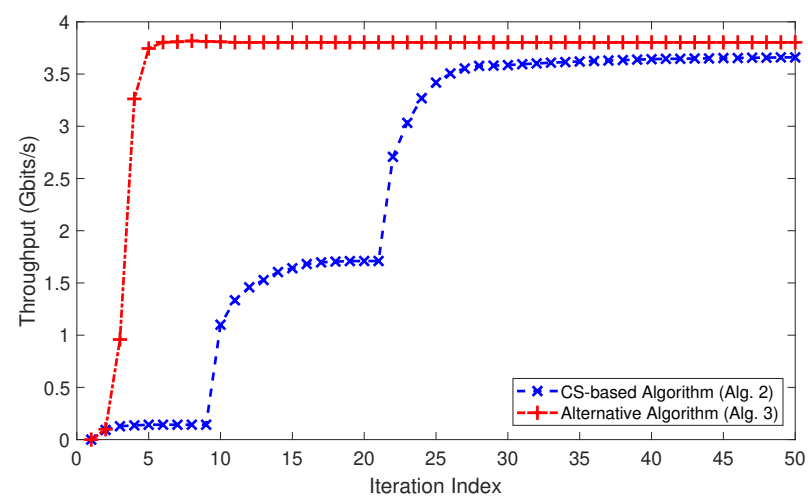

Fig. 4. The achieved throughput versus the iteration indices.

We illustrate the convergence of our proposed algorithms in Fig. 4 where the variations in the total throughput achieved by Algorithm 2 and 3 over the iterations are shown. As can be seen, both algorithms adapt well with the system setting and return the effective solution where the throughput of each approach increases over the iterations before saturating at its maximum value. In addition, Algorithm 2 requires a higher number of iterations to converge due to its two-loop process in which the inner loop is employed to find the optimal power allocation and RU assignment for given MCS indices while the outer loop stands for adjusting the MCS selection. The figure also demonstrates the superiority of Algorithm 3 where this algorithm achieves a slightly better system throughput and converges faster. The provided complexity analysis in Section $\mathrm{V}-\mathrm{A}$ shows that the alternative algorithm requires a higher computation complexity than the CS-based algorithm. Specifically, the complexity of Algorithm 2 is $O\left(I_{\mathrm{alg} 2} L_{\mathrm{RU}} N \zeta_{2}^{-2}\right)$ while the that of Algorithm 3 is $O\left(I_{\mathrm{alg} 3}\left(N+L_{\mathrm{RU}}\right)^{2} N L_{\mathrm{RU}} \log \left(N+L_{\mathrm{RU}} C_{\mathrm{SC}}\right)\right)$. Taking into account that $N+L_{\mathrm{RU}}=7+65=72$ and the numbers of iterations required for for Algorithms 2 and 3 to converge are roundly 40 and 10 , respectively, one can conclude the higher complexity of Algorithm 3 in this simulation. This explains why Algorithm 3 can outperforms Algorithm 2 due to the normal trade-off between the complexity and performance.

Fig. 5 presents the throughput versus the maximum transmit power of AP, $P_{\mathrm{T}}$, for two proposed algorithms. For comparison purposes, the results achieved by employing the supercomputing servers on NEOS website for solving problem (22), named "Neos Server", are added as the optimal solution (or near to optimal solution). Moreover, we also demonstrate the throughput achieved by Wang's Alg. presented in Section V-B and two other simple schemes, i.e. the fixed MCS scheme and unicast transmission scheme. Here, the modulation and coding

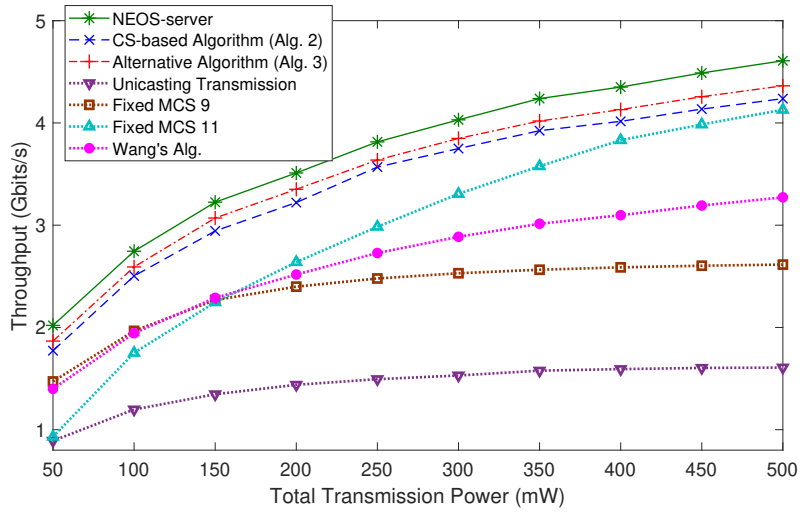

Fig. 5. The achieved throughput versus the power transmission budget.

rate in the fixed MCS scheme are kept unchanged for all UUs and MGs. Then, the throughput of this scheme is obtained by exploiting Algorithm 3 without updating $m$ in Step 6. In this figure, we plot two curves corresponding to this scheme with two fixed MCS indices 9 and 11, namely 'fixed MCS 9' and 'fixed MCS 11', respectively. On the other hand, in the unicast transmission scheme, we assume that all users in the MGs are the unicast users where each of them requires a separate RU for data transmission. Again, Algorithm 3 is also implemented to achieve the throughput of this scheme and its corresponding curve is labelled 'Unicasting Transmission' in the figure. It is observed that the throughput achieved by every scheme increases with $P_{\mathrm{T}}$ which is due to the fact that larger feasible sets can attain better solutions. The figure also shows that Algorithm 3 achieves a slightly higher achievable throughput than Algorithm 2 over all the values of $P_{\mathrm{T}}$. Additionally, our proposed algorithms outperform Wang's Alg. and other simple schemes with unicast transmission or fixed MCS assignment. This confirms the advantage of utilizing multicast transmission integrating with link adaptation strategy. Interestingly, the results obtained by NEOS-server provide the highest throughput; however, it illustrates that the gaps between our proposed algorithms and the optimum results are quite limited.

Fig. 6 illustrates the minimum, maximum, and mean MCS indices selected for all UUs/MGs in four schemes, "Neos Server", Wang's Alg., and our proposed solutions with Algorithms 2 and 3 versus the maximum transmit power of AP, $P_{\mathrm{T}}$. It is observed that the higher MCS indices are selected by all schemes due to the higher transmit power budget. In this context, the higher MCS index selection results in the increase of the achieved throughput which is illustrated in the Fig. 5. This figure also shows that all four schemes return different MCS indices for UUs/MGs. Specifically, "Neos Server" chooses the 


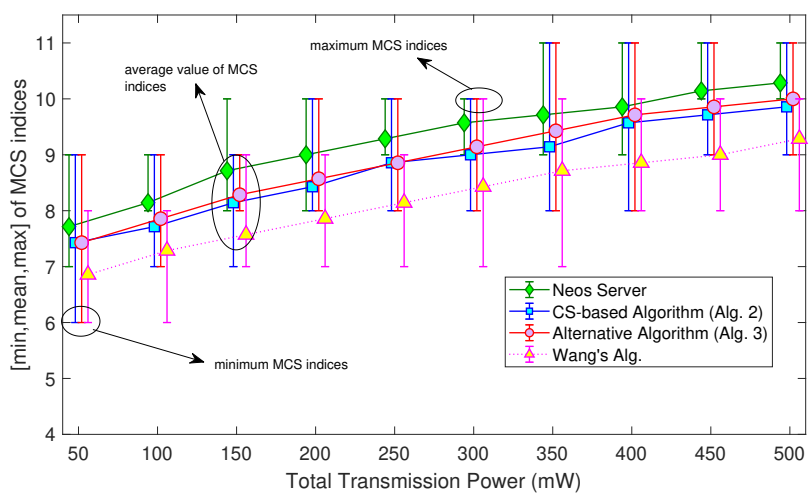

Fig. 6. The minimum, mean, and maximum MCS indices selected for all users versus the power transmission budget.

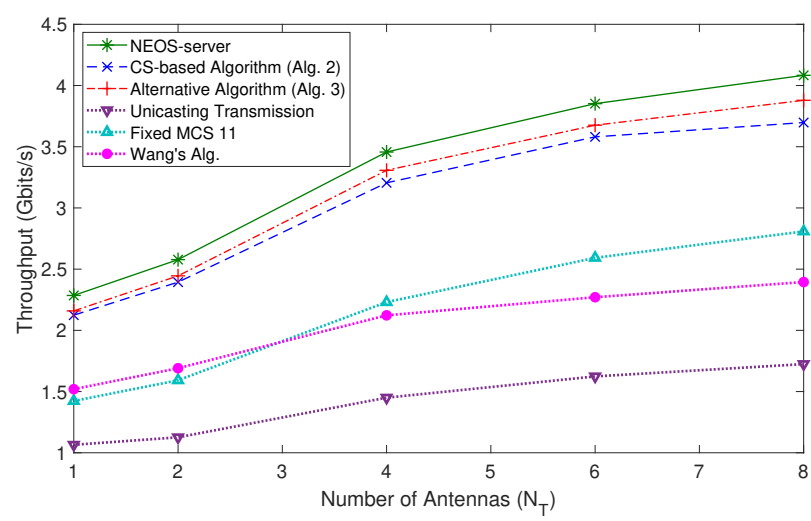

Fig. 7. The achieved throughput versus the number of antennas.

better MCS indices for UUs/MGs while Wang's Alg. selects the lower MCS indices comparing to our proposed algorithms. For instance, when $P_{\mathrm{T}}=250 \mathrm{~mW}$, the chosen MCS indices of "Neos Server" vary from 9 to 10, that of Wang's Alg. are in the range of $[7,9]$, while our schemes, i.e., Algorithm 2 and 3 select the same MCS range of $[8,10]$. Moreover, beside showing that "Noes Server" returns the highest mean of selected MCS indices, Fig. 6 also demonstrates the superior performance of our proposed algorithms compared to Wang's Alg. since they enhance the wireless resources and provide the higher-rate MCS selection for users' transmission.

The benefits of multiple-antenna transmission are shown in Fig. 7 where we plot the throughput versus number of antennas employed at the AP for six schemes: two proposed algorithms, 'NEOS server', Wang's Alg.', 'Unicasting Transmission', and 'fixed MCS 11'. As expected, the larger numbers of equipped antennas result in the higher system throughput due to the increase in the degrees of freedom for the precoder design. In addition, our proposed algorithms can achieve throughput outcomes close to those obtained by the NEOS optimization solver. Moreover, this figure also confirms the superiority of Algorithms 2 and 3 where significant gaps are observed between the throughput of the proposed algorithms and that obtained by Wang's Alg., the unicast transmission and fixed MCS assignment schemes. Once again, the alternative algo-

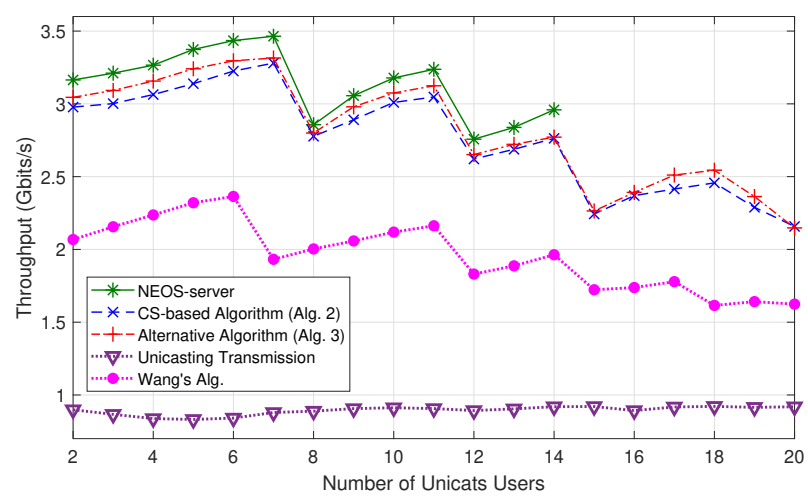

Fig. 8. The achieved throughput versus the number of UUs.

rithm slightly outperforms the iterative CS-based algorithm.

In Fig. 8, we present the system throughput versus the number of UUs in the network. Here, we sequentially add more users to obtain different points on each curve. As a reference, the throughput results achieved by Wang's Alg. and the unicast transmission scheme are also illustrated. Interestingly, the system throughput trend goes down when the number of UUs increases. Although the system throughput slightly increases over short segments, it drops down significantly at some specific points. This is due to the fact that our design aims to allocate to every $\mathrm{UU}$ or MG one RU for data transmission. Hence, the bandwidth in our system needs to be channelized such that the number of available RUs is greater than or equal to the number of UUs/MGs. Then, adding more UUs results in a re-channelization and a RU re-allocation, which may causes a throughput decrease. Moreover, to maximize the throughput, a large-bandwidth $\mathrm{RU}$ is normally assigned to MGs consisting of many MUs. Therefore, the more UUs the network serves, the lower the chance that the such good RUs are assigned to MGs. This can also make the throughput drop as illustrated in the figure. Again, our designed schemes achieve the higher throughput than "Wang's Alg." while the NEOS server outperforms ours. The alternative algorithm can return a slightly better solution than the iterative CS-based algorithm; however, the difference between the these two algorithms is very limited. In addition, the figure demonstrates that the schemes which are designed to serve coexisting unicast and multicast users can obtain significantly higher throughputs than the unicast transmission scheme.

Next, we evaluate the system performance in the open space by utilizing the mode $\mathbf{F}$ of WiFi channel model to generate the channel vectors [42]. A possible use case of this scenario is a group of public safety officers leveraging 802.11ax multicast services in a disaster recovery area. In this simulation, we randomly locate the users in different zones with different distances to the AP. Specifically, every zone refers to as a ring whose minimum and maximum radii are $d_{\min }$ and $d_{\max }(\mathrm{m})$ as illustrated in Fig. 3, respectively. Here, there are five zones are selected for user allocation where the values of $\left[d_{\min }, d_{\max }\right]$ corresponding to the five zones are $[10,50]$, 


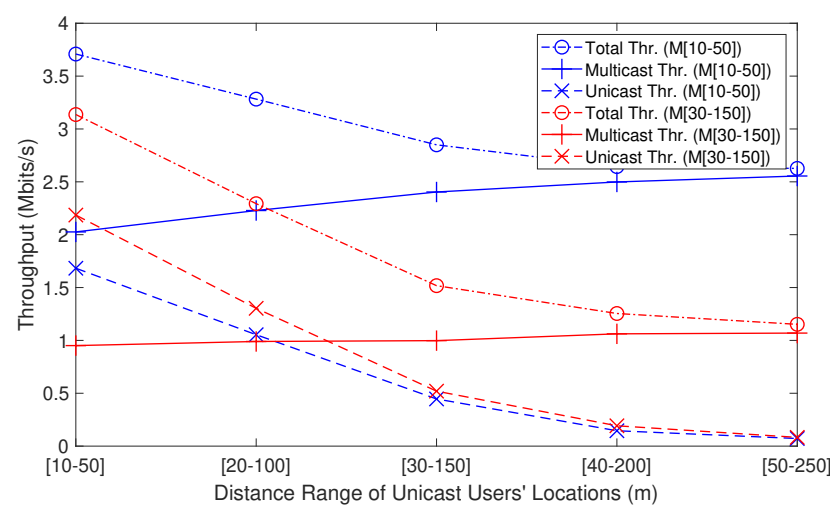

Fig. 9. The achieved throughput versus the distance range of UUs.

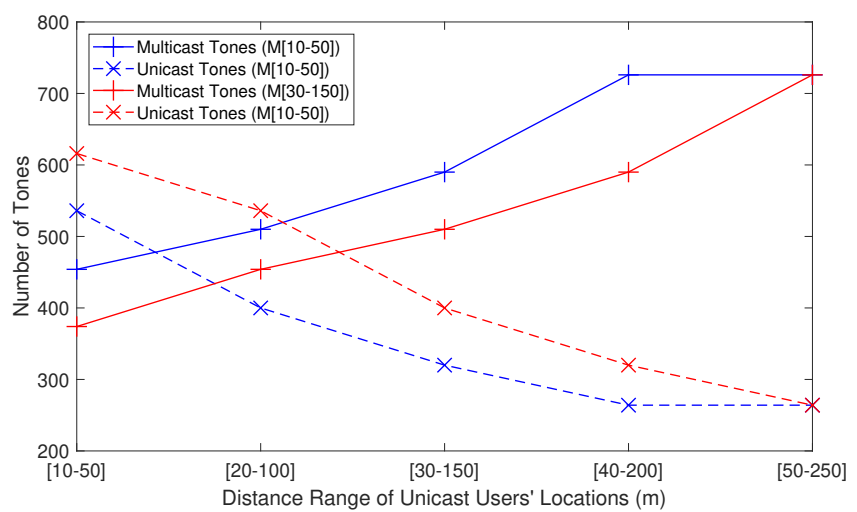

Fig. 10. The number of tones assigned to the MGs and UUs versus the distance range of UUs.

$[20,100],[30,150],[40,200]$, and $[50,250]$, respectively. The maximum transmission power of the AP in this simulation is set at $200 \mathrm{~mW}\left(P_{\mathrm{T}}=200 \mathrm{~mW}\right)$. To obtain the throughput, we employ Algorithm 3 due to its significant performance. Fig.s 9 and 10 illustrate the system throughput and the number of tones assigned to UUs/MGs versus the various location zones of the UUs. In this simulation, the MGs are randomly placed in two different zones, i.e. [10,50] and [30,150]. It is observed that as the distances between the AP and the UUs (or MGs) increases, the throughput obtained by the MGs (or UUs) increases while that due to the UUs (or MGs) decreases. In addition, when some devices (UUs or users in MGs) are placed far away from the AP, the total system throughput also decreases. These results are due to the fact when the links of some devices degrade, the performance of the whole network becomes lower. In such scenarios, the system decides to allocate more radio resources to the remaining devices with stronger links to maximize the total throughput, as shown in Fig. 10

\section{CONCLUSION}

In this paper, we have proposed novel JRRMLA designs maximizing the system throughput of the 802.11ax based WLAN systems serving coexisting unicast and multicast transmission. Specifically, we have proposed a two-stage solution framework which can determine the precoders' directions in stage one and completely design the channelization, RU assignment, and MCS selection in stage two. To fulfil the design goals in these two stages, two precoder design mechanisms for unicast and multicast transmission together with two efficient joint RRM and LA algorithms are developed. The numerical results have confirmed the significant performance gains obtained by the proposed algorithms where they were shown to achieve a throughput close to the near-optimal solution given by the super computers of NEOS servers. In addition, the proposed algorithms significantly outperform the benchmark solution given in literature and other schemes with fixed MCS assignment and only-unicast users. In future works, we plan to extend our designs to mesh networks consisting of multiple APs and dense accessing users of multiple mesh 802.11ax stations with routing strategy as an alternative parameter of joint optimization. Employing reinforcement learning to help the system respond quickly to the variations in the channel gains and the movement of users is also a potential future direction.

\section{APPENDIX A \\ PROOF OF PROPOSITION 1}

Since the SNR of UU $u$ over tone $t$ of $R U(\ell, i)$ mainly depends on the channel vector between the user and the AP, the best precoder for this transmission can be designed as the conjugate beamforming vector, i.e,

$$
\mathbf{w}_{\ell, i, t}^{u}=\kappa \ell, i, t \mathbf{h}_{\ell, i, t}^{u}
$$

where $\kappa_{\ell, i, t}$ is a scalar. Due to the power constraint on all tones of $R U(\ell, i)$, the optimal temporary precoder for $\mathrm{UU} u$ can be determined as

$$
\mathbf{w}_{\ell, i, t}^{u, \text { temp }}=\sqrt{p_{\ell, i, t}^{u}} \mathbf{h}_{\ell, i, t}^{u} \quad \forall t \in \mathcal{S}_{(\ell, i)},
$$

where $p_{\ell, i, t}^{u}$ can be considered as the power share of tone $t$. Substituting (43) into problem (12) and exploiting (8), one can reform the problem in (12) as follows

$$
\begin{array}{cc}
\min _{\left\{p_{\ell, i, t}^{u}\right\}^{\prime} s} & \sum_{t \in \mathcal{T}_{(\ell, i)}} \exp \left(-p_{\ell, i, t}^{u} \bar{\gamma}_{\ell, i, t}^{\mathrm{uni}, u}\right) \\
\text { s.t. } & \sum_{t \in \mathcal{S}_{(\ell, i)}}\left|\mathbf{h}_{\ell, i, t}^{u}\right|^{2} p_{\ell, i, t}^{u} \leq 1 .
\end{array}
$$

As can be observed, this problem is convex; hence, its optimal solution can be obtained by studying the Karush-Kuhn-Tucker optimality conditions. The Lagrangian of the problem (44) can be expressed as

$$
\mathcal{L}\left(\mathbf{p}^{u}, \mu\right)=\sum_{t \in \mathcal{T}_{(\ell, i)}} e^{-p_{t}^{u} \bar{\gamma}_{\ell, i, t}^{\text {uni, } u}}+\mu\left(\sum_{t \in \mathcal{S}_{(\ell, i)}}\left|\mathbf{h}_{\ell, i, t}^{u}\right|^{2} p_{\ell, i, t}^{u \star}-1\right),
$$

where $\mu$ is the Lagrangian multiplier associated with the power constraint 44b and $\mathbf{p}_{\ell, i}^{u}$ stands for all variables $p_{\ell, i, t}^{u}$. In addition, the dual function of problem (44) can be written as

$$
g(\mu)=\max _{\mathbf{p}_{\ell, i}^{u}} \mathcal{L}\left(\mathbf{p}_{\ell, i}^{u}, \mu\right) .
$$


TABLE V

SUMMARY OF KEY NOTATIONS

\begin{tabular}{|c|c|}
\hline Notations & Description \\
\hline$a_{\rho, i}^{v}$ & Assignment variable for UU/MG $v$ and $R U(\ell, i)$ \\
\hline $\mathbf{b}_{\ell, i}$ & Overlap vector between $R U(\ell, i)$ and the 26 -tone RUs, defined in $(1)$ \\
\hline$B L E R(m, \gamma)$ & BLER corresponding to $m$ and $\gamma$ \\
\hline$c_{\mathrm{C}}(m)$ & Modulated bits per symbol and channel coding rate for MCS index $m$ \\
\hline$d_{\min }$ and $d_{\max }$ & Minimum and maximum radii of the ring in which users are located \\
\hline $\mathcal{G}^{\mathrm{MG}}$ & Set of all MGs \\
\hline $\mathcal{G U}$ & Set of all UUs and MGs, $\mathcal{G} \mathcal{U}=\mathcal{U}^{\mathrm{UU}} \cup \mathcal{G}^{\mathrm{MG}}$ \\
\hline $\mathbf{h}_{\ell, i, t}^{\mathrm{x}}$ & Channel vector between the AP and user $\mathrm{x}$ over tone $t$ of $R U(\ell, i)$ \\
\hline$I_{\mathrm{alg} 2}, I_{\mathrm{alg} 3}$ & Number of iterations for implementing Algorithm 2 and 3 respectively \\
\hline$L^{v}, O^{v}$ & Packet and the overhead lengths (in bits) corresponding to UU/MG $v$ \\
\hline$L_{\mathrm{lw}}$ & Penetration loss for a single wall \\
\hline$m^{v}$ & MCS index corresponding to UU/MG $v$ \\
\hline$M_{\mathrm{RU}}$ & Number of all RUs \\
\hline$N^{\mathrm{UU}}, N^{\mathrm{MU}}, N^{\mathrm{MG}}$ & Numbers of UUs, MUs, and MGs, respectively \\
\hline$N_{\top}$ & Number of antennas equipped at the AP \\
\hline$n_{\mathrm{w}}$ & Number of walls penetrated \\
\hline$p_{\ell, i}^{v}$ & power allocation for UU/MG $v$ over $R U(\ell, i)$ \\
\hline$P_{\mathrm{T}}$ & Maximum transmission power of the AP \\
\hline$P L_{\text {overall }}$ & Overall path loss \\
\hline $\begin{array}{l}P L_{\text {indoor }}(d) \\
P E L_{\text {wall }}\end{array}$ & $\begin{array}{l}\text { Estimated as } 20 \log _{10}(d) \text { if } d \leq d_{\mathrm{BP}} \text { and } 35 \log _{10}(d)-15 \log _{10}\left(d_{\mathrm{BP}}\right) \text { if } d>d_{\mathrm{BP}} \text {, } \\
\text { Wall penetration }\end{array}$ \\
\hline$P E L_{\text {shadow }}$ & Shadow fading loss \\
\hline$q_{\ell, i}^{v}$ & Weight corresponding to the one-to-one matching between $R U(\ell, i)$ and UU/MG $v$ \\
\hline$r_{\mathrm{cr}}(m)$ & Channel coding rate for MCS index $m$ \\
\hline$S_{\ell, i}$ & Number of tones in $R U(\ell, i)$ \\
\hline $\mathcal{S}_{\ell, i}$ & Set of tones in $R U(\ell, i)$ \\
\hline & Transmitted symbol corresponding to UU/MG $v$ and tone $t$ \\
\hline$T_{\ell, i}^{\mathrm{x}}\left(m^{v}, \gamma_{\ell, i}^{\mathrm{x}}\right)$ & Throughput of user $\mathrm{x}$ over $R U(\ell, i)$ corresponding to $m^{v}$ and $\gamma_{\ell, i}^{\mathrm{x}}$ \\
\hline$R U(\ell, i)$ & RU corresponding to splitting level $\ell$ and position $i$ \\
\hline $\mathcal{U}_{n}^{\mathrm{mul}}$ & the set of MUs in MG $n$ \\
\hline $\mathcal{U}^{\mathrm{UU}}$ & Set of all UUs \\
\hline $\mathbf{w}_{\ell, i}^{v}$ & Precoding vector designed for UU/MG $v$ over tone $t$ of $R U(\ell, i)$ \\
\hline $\mathbf{w}_{\ell, i, t}^{\mathrm{x}, \text { temp }}$ & Temporary precoder of user $\mathrm{x}$ over tone $t$ of $R U(\ell, i)$ \\
\hline$X_{\mathrm{UU}-\mathrm{BF}}$ & Complexity of designing precoders for UUs \\
\hline$X_{\mathrm{MG}-\mathrm{BF}}$ & Complexity of designing precoders for MGs \\
\hline$X_{\mathrm{alg} 2}, X_{\mathrm{alg} 2}$ & Complexity of Algorithm 2 and 3 respectively \\
\hline$z_{\ell, i, t}^{\mathrm{x}}$ & Noise vector at user $\mathrm{x}$ over tone $t$ of $R U(\ell, i)$ \\
\hline$\alpha(m)$ & Slope value of BLER formula corresponding to MCS index $m$ \\
\hline$\beta(m)$ & Dropping point of BLER formula corresponding to MCS index $m$ \\
\hline $\bar{\gamma}_{\ell, i}^{\mathrm{x}}$ & Effective SNR of user $\mathrm{x}$ over $R U(\ell, i)$ obtained in stage one \\
\hline$\zeta(m)$ & EESM setting parameter related to MCS index $m$ \\
\hline
\end{tabular}

Due to the convexity of the objective function, the problem can be solved by using the KKT condition $\partial \mathcal{L}\left(\mathbf{p}_{\ell, i}^{u}, \mu\right) / \partial p_{\ell, i, t}^{u}=0$, which is equivalent to

$$
p_{\ell, i, t}^{u, \prime}=-\frac{1}{\bar{\gamma}_{\ell, i, t}^{\mathrm{uni}, u}} \ln \left(\mu\left|\mathbf{h}_{\ell, i, t}^{H, u}\right|^{2} / \bar{\gamma}_{\ell, i, t}^{\mathrm{uni}, u}\right),
$$

In addition, the optimal solution of $p_{\ell, i, t}^{u, \prime}$ must satisfy constraint (44b). In addition, the objective function is a decreasing function with respect to $\mathbf{p}_{\ell, i}^{u}$; hence, the power constraint 44b must be met with equality. Therefore, $\mu$ can be determined to satisfy $\sum_{t \in \mathcal{S}_{(\ell, i)}}\left|\mathbf{h}_{\ell, i, t}^{u}\right|^{2} p_{\ell, i, t}^{u, \prime}=1$. This completes the proof of Proposition 1

\section{APPENDIX B}

PROOF OF PROPOSITION 2

Problem 28 can be solved optimally by studying the Karush-Kuhn-Tucker conditions. Its Lagrangian function can be expressed as

$$
\begin{aligned}
& \mathcal{L}(\mathbf{p}, \rho)=\sum_{\forall(\ell, i)}\left[\sum_{n \in \mathcal{G}^{\mathrm{MG}}} a_{\ell, i}^{n} \omega_{\ell, i}^{n} \sum_{k \in \mathcal{U}_{n}^{\mathrm{mul}}} e^{-\alpha_{\ell, i}^{n, k} p_{\ell, i}^{n}}\right. \\
& \left.+\sum_{u \in \mathcal{U}^{\mathrm{UU}}} a_{\ell, i}^{u} \omega_{\ell, i}^{u} e^{-\alpha_{\ell, i}^{u} p_{\ell, i}^{u}}\right]+\rho\left(\sum_{\forall(\ell, i)} \sum_{\nu \in \mathcal{G} \mathcal{U}} p_{\ell, i}^{v}-P_{\mathrm{T}}\right),
\end{aligned}
$$

where $\rho$ is the Lagrangian multiplier. The dual function of problem $28 p$ can be written as $g(\rho)=\max _{\mathbf{p}} \mathcal{L}(\mathbf{p}, \mu)$. Here, we have

$$
p_{\ell, i}^{v *}=0 \text { if } a_{\ell, i}^{v}=0 .
$$

On the other hand, when $a_{\ell, i}^{v}=1, p_{\ell, i}^{v}$ can be determined by taking the derivative of $\mathcal{L}(\mathbf{p}, \rho)$ and then letting the results equal to zero, i.e., $\partial \mathcal{L}(\mathbf{p}, \rho) / \partial p_{\ell, i}^{v}=0$ yields

$$
\begin{aligned}
p_{\ell, i}^{u *} & =\frac{1}{\alpha_{\ell, i}^{u}} \ln \left(\omega_{\ell, i}^{u} \alpha_{\ell, i}^{u} / \rho\right), \forall u \in \mathcal{U}^{\mathrm{UU}}, \\
p_{\ell, i}^{n * r} & =\left.p^{\prime}\right|_{\ell_{\ell, i}^{n}\left(p^{\prime}\right)=\rho}, \forall n \in \mathcal{G}^{\mathrm{MG}},
\end{aligned}
$$


In addition, the objective function is a decreasing function with respect to $\mathbf{p}$; hence, the power constraint must be met with equality. Therefore, $\rho$ must be chosen so that $\sum_{v \in \mathcal{G} \mathcal{U}} \sum_{\forall(\ell, i)} p_{\ell, i}^{v *}$ equals to $P_{\mathrm{T}}$ where $p_{\ell, i}^{v \star}$ is determined according to $\rho$ as in (49)-(51).

\section{REFERENCES}

[1] D. Evans, "The internet of things: How the next evolution of the internet is changing everything," CISCO White Paper, Apr. 2011.

[2] E. D. Ngangue Ndih and S. Cherkaoui, " On enhancing technology coexistence in the IoT era: ZigBee and 802.11 Case," IEEE Access 4, pp. 1835-1844, May 2016.

[3] E. Khorov, A. Kiryanov, A. Lyakhov, and G. Bianchi, "A tutorial on ieee 802.11ax high efficiency wlans," IEEE Communications Surveys Tutorials, vol. 21, no. 1, pp. 197-216, Firstquarter 2019.

[4] D.J. Deng, Y.P. Lin,X. Yang,J. Zhu,Y.B. Li,J. Luo, and K.C. Chen, "IEEE 802.11 ax: highly efficient WLANs for intelligent information infrastructure" IEEE Communications Magazine vol. 55, no. 12, pp. 52-59, Dec 2017.

[5] "Ieee p802.11 task group ax," High Efficiency (HE) Wireless LAN Task Group, Tech. Rep. [Online]. Available: http://www.ieee802.org/11/ Reports/tgaxupdate.htm

[6] B. Bellalta, "Ieee 802.11ax: High-efficiency wlans," IEEE Wireless Communications, vol. 23, no. 1, pp. 38-46, February 2016.

[7] WiFi Alliance, "Wi-Fi 6: Advanced uses for new ear of connectivity," White Paper, pp. 1-10, Oct. 2019.

[8] A. Z. Yalcin and M. Yuksel, "Precoder design for multi-group multicasting with a common message," IEEE Transactions on Communications, vol. 67 , no. 10 , pp. 7302-7315, Oct. 2019.

[9] "The Wi-Fi Market and the Genesis of 802.11ax," Aruba Networks White Paper, 2018. [Online]. Available: https://www.arubanetworks. com/assets/wp/WP_802.11AX.pdf

[10] E. Chiu, V. K. N. Lau, "Precoding design for multi-antenna multicast broadcast services with limited feedback," IEEE Systems J., vol. 4, no. 4, pp. 550-560, Dec. 2010.

[11] J. Lee and C. Kim, "An efficient multiple access coordination scheme for OFDMA WLAN,” IEEE Communications Letters, vol. 21, no. 3, pp. 596-599, March 2017.

[12] E. Avdotin, D. Bankov, E. Khorov, and A. Lyakhov, "OFDMA resource allocation for real-time applications in IEEE 802.11ax networks," 2019 IEEE International Black Sea Conference on Communications and Networking (BlackSeaCom), 2019.

[13] D. Bankov, A. Didenko, E. Khorov, and A. Lyakhov, "OFDMA uplink scheduling in IEEE 802.11ax networks," 2018 IEEE International Conference on Communications (ICC), 2018.

[14] K. Wang, and K. Psounis, "Scheduling and resource allocation in 802.11ax," IEEE INFOCOM 2018 - IEEE Conference on Computer Communications, 2018.

[15] K. Lee, "Using OFDMA for MU-MIMO user selection in 802.11axbased Wi-Fi networks," IEEE Access, vol. 7, pp. 186041-186055, 2019.

[16] L. Li, G. Oikonomou, M. Beach, R. Nejabati and D. Simeonidou, "An SDN agent-enabled rate adaptation framework for WLAN," 2019 IEEE International Conference on Communications (ICC), 2019.

[17] R. Karmakar, S. Chattopadhyay and S. Chakraborty, "Intelligent MUMIMO user selection with dynamic link adaptation in IEEE 802.11ax," IEEE Transactions on Wireless Communications, vol. 18, no. 2, pp 1155-1165, Feb. 2019.

[18] I. Osunkunle, "AL-FEC Wireless Rate Adaptation for WiFi Multicast," 2018 IEEE Canadian Conference on Electrical \& Computer Engineering (CCECE), 2018.

[19] S. Choudhury and J. D. Gibson, "Payload length and rate adaptation for multimedia communications in wireless LANs," IEEE Journal on Selected Areas in Communications, vol. 25, no. 4, pp. 796-807, May 2007.

[20] A. Sahai and P. Grover, "The price of certainty: "waterslide curves" and the gap to capacity," arXiv preprint, arXiv:0801.0352, Jan. 2008.

[21] S. Park, R. C. Daniels, and R. W. Heath, "Optimizing the target error rate for link adaptation" in Proc. IEEE Global Communications Conference (GLOBECOM), pp. 1-6, Dec. 2015.

[22] "Feasibility study for OFDM for UTRAN enhancement, Release 6," 3rd Generation Partnership Project, Technical Document, TSG-RAN TR25.892 v1.1.0, Mar. 2004

[23] "System-level evaluation of OFDM-Further considerations," document 3GPP TSG-RAN-1. R1-031303, Portugal, 2003.
[24] Z. Hanzaz and H. Schotten, "Analysis of effective SINR mapping models for MIMO OFDM in LTE system," in Proc. Int. Wireless Commu. and Mobile Comp. Conf., July 2013, pp. 1509-1515.

[25] K. Brueninghaus et al., "Link performance models for system level simulations of broadband radio access systems," in IEEE 16th International Symposium on Personal, Indoor and Mobile Radio Communications, Berlin, 2005, pp. 2306-2311.

[26] "Spatial channel model for MIMO simulations" document 3GPP-TSGRAN TR-25.996 v6.1.0, Sept. 2003.

[27] I. Necoara, A. Patrascu, "Iteration complexity analysis of dual first order methods for conic convex programming" Optimization Methods \& Software, vol. 31, no. 3, pp. 1-34, Mar. 2016.

[28] M. Grant and S. Boyd, "CVX: Matlab software for disciplined convex programming, version 2.1," Online: http://cvxr.com/cvx, Mar. 2014.

[29] E. Dolan, "The NEOS Server 4.0 Administrative Guide," Technical Memorandum ANL/MCS-TM-250, Mathematics and Computer Science Division, Argonne National Laboratory, 2001.

[30] E. Candes, M. Wakin, and S. Boyd, "Enhancing sparsity by reweighted $\ell_{1}$ minimization," J. Fourier Analysis Applications, vol. 14, no. 5, pp. 877-905, Dec. 2008.

[31] J. Zhao, T. Q. S. Quek, and Z. Lei, "Coordinated multipoint transmission with limited backhaul data transfer," IEEE Trans. Wireless Commun., vol. 12, no. 6, pp. 2762-2775, Jun. 2013.

[32] B. Dai and W. Yu, "Sparse beamforming and user-centric clustering for downlink cloud radio access network," IEEE Access, vol. 2, pp. 13261339, Oct. 2014.

[33] G. Caron, P. Hansen, B. Jaumard, "The assignment problem with seniority and job priority constraints," Operations Research, vol. 47, no. 3, pp. 449-453, Jun. 1999.

[34] U. Bauer, “Assignment Problem with Constraints," Master's thesis, ETH Zurich Department of Computer Science, 2004.

[35] The MathWorks, Inc., "802.11ax Compressed Beamforming Packet Error Rate Simulation [Online]", 2020. Available at: https://www.mathworks.com/help/wlan/examples/ 802-11ax-compressed-beamforming-packet-error-rate-simulation.html

[36] Jianhan, L., Ron, P. et al., "TGax channel model" IEEE 802.1114/0882r4, Sept. 2014.

[37] P. Poshala, K. K. Rushil, R. Gupta, "Signal chain noise figure analysis", in Application Report - Texas Instrument, no. SLAA652, Oct. 2014.

[38] D. P. Palomar and J. R. Fonollosa, "Practical algorithms for a family of waterfilling solutions", IEEE Trans. Signal Process., vol. 53, no. 2, pp. 686-695, Feb. 2005.

[39] A. Ben-Tal and A. Nemirovski, Lectures on Modern Convex Optimization: Analysis, Algorithms, and Engineering Applications, ser. MPSSIAM Series on Optimization. Philadelphia, Pennsylvania: Society for Industrial and Applied Mathematics, 2001.

[40] C. Cartis, N. I. M. Gould, and P. H. L. Toint, "On the complexity of steepest descent, Newton's and regularized Newton's methods for nonconvex unconstrained optimization problems," SIAM J. Optim., vol. 20, no. 6, pp. 2833-2852, Oct. 2010.

[41] Ulrich Bauer, Assignment problem with constraints. Master's thesis, ETH Zurich Department of Computer Science, 2005.

[42] J. Liu, R. Porat, N. Jindal, and et al., "IEEE 802.11ax channel model document," IEEE 802.11 document 802.11-14/0882r4, Sept. 2014.

[43] P. Huang et al., "MU-RTS/CTS for DL MU". Access on Nov. 24, 2020 [Online]. Available: https://mentor.ieee.org/802.11/dcn/15/11-150867-01-00axmu-rts-cts-for-dl-mu.pptx.

[44] P. M. Olmos and R. Urbanke, "A scaling law to predict the finiteLength performance of spatially-coupled LDPC codes," IEEE Trans. Inf. Theory, vol. 61, no. 6, pp. 3164-3184, June 2015.

[45] D. Jungnickel, chapter "Weighted matchings," in Graphs, Networks and Algorithms, Springer Berlin Heidelberg, vol. 5, pp. 419-456, 2008.

[46] S. C. Chapra and R. P. Canale, Numerical methods for engineers, seventh edition. Mc. Graw Hill, 2015.

[47] C. Arkenberg, P. Giorgio, and C. Deweese, "Redesigning stadiums for a better fan experience". Access on Jan. 13, 2021 [Online]. Available: https://www2.deloitte.com/us/en/insights/industry/telecommunications/instadium-fan-experience.html. 


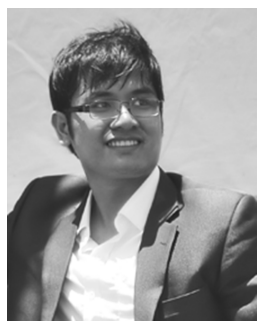

Vu Nguyen Ha (S'11-M'17) received the B.Eng. degree (with honors) from the French training program for excellent engineers in Vietnam (PFIEV), Ho Chi Minh City University of Technology (HCMUT), Vietnam and an addendum degree from de École Nationale Supérieure des Télécommunications de Bretagne-Groupe des École des Télécommunications, Bretagne, France in 2007. From 2008 to 2011, he was a Research Assistant with the School of Electrical Engineering, University of Ulsan, Ulsan, Korea. He received the Ph.D. degree (with honors and innovation award) from the Institut National de la Recherche Scientifique-Énergie, Matériaux et Télécommunications (INRS-EMT), Université du Québec, Montréal, Québec, Canada in 2017. From 2016 to 2019 , he worked as a postdoctoral fellow at École Polytechnique de Montréal, Montréal, Québec, Canada. Currently, he holds the same role at the Resilient Machine learning Institute (ReMI) of the École de Technologie Supérieure (ÉTS), University of Québec, Montréal, Canada. His research interests include radio resource management and emerging enabling technologies for 5G wireless systems with special emphasis on Heterogeneous Small-cell Networks, Cloud RAN, massive MIMO communications, Mobile Egde Computing, and 802.11ax WiFi. He was a recipient of the FRQNT Post-doctoral Fellowship for International Researcher (PBEEE) awarded by Québec Ministry of Education in 2018 and 2019. In 2021, he was also awarded a Certificate for Exemplary Reviews by the IEEE Wireless Communications Letters.

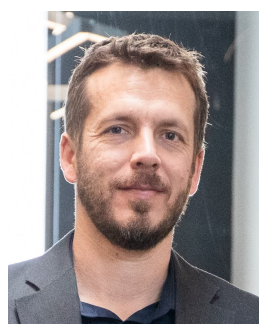

Dr. Gwenael Poitau has over 18 years of experience developing wireless communication systems for the telecom and defense markets. He began his career with Wavesat in Montréal developing signal processing algorithms and ASIC architectures for $4 \mathrm{G}$ chipsets. He was then promoted as technical lead for LTE development. He continued to work on LTE at Interdigital where he generated Intellectual Property on Device-to-Device communications and participated to LTE Rel. 12 standardization. He joined Ultra Electronics TCS in July 2010 as Wireless Architect for the new ORION radio product line and was promoted Chief Technology Officer in September 2013. In addition to its CTO role he took the Head of Engineering position in 2016. In 2019, he co-founded the Resilient Machine learning Institute, in collaboration with the École de Technologie Supérieure (Montréal), to enhance the resilience of critical communications systems with distributed AI/ML techniques. Gwenael holds PhD, Master and Bachelor degrees in Physics \& Electrical Engineering from Telecom SaintEtienne and INSA Lyon (France). He has published a large number of patents and scientific articles in wireless technology and now specializes in multiagent machine learning.

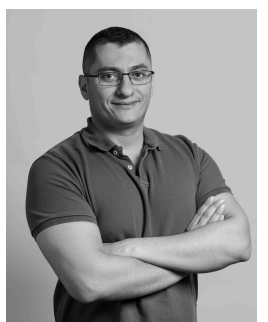

Georges Kaddoum (M'11-SM'20) received the Bachelor's degree in electrical engineering from the École Nationale Supérieure de Techniques Avancées (ENSTA Bretagne), Brest, France, and the M.S degree in telecommunications and signal processing(circuits, systems, and signal processing) from the Université de Bretagne Occidentale and Telecom Bretagne (ENSTB), Brest, in 2005 and the Ph.D. degree (with honors) in signal processing and telecommunications from the National Institute of Applied Sciences (INSA), University of Toulouse, Toulouse, France, in 2009. He is currently an Associate Professor and Tier 2 Canada Research Chair with the École de Technologie Supérieure (ÉTS), Université du Québec, Montréal, Canada. In 2014, he was awarded the ÉTS Research Chair in physical-layer security for wireless networks. Since 2010, he has been a Scientific Consultant in the field of space and wireless telecommunications for several US and Canadian companies. He has published over 200+ journal and conference papers and has two pending patents. His recent research activities cover mobile communication systems, modulations, security, and space communications and navigation. Dr. Kaddoum received the Best Papers Awards at the 2014 IEEE International Conference on Wireless and Mobile Computing, Networking, Communications (WIMOB), with three coauthors, and at the 2017 IEEE International Symposium on Personal Indoor and Mobile Radio Communications (PIMRC), with four coauthors. Moreover, he received IEEE Transactions on Communications Exemplary Reviewer Award for the year 2015, 2017, 2019. In addition, he received the research excellence award of the Université du Québec in the year 2018. In the year 2019, he received the research excellence award from the ÉTS in recognition of his outstanding research outcomes. Prof. Kaddoum is currently serving as an Associate Editor for IEEE Transactions on Information Forensics and Security, and IEEE Communications Letters. 